\title{
The Symmetry Group of Lamé's System and the Associated Guichard Nets for Conformally Flat Hypersurfaces ${ }^{\star}$
}

\author{
João Paulo dos SANTOS and Keti TENENBLAT \\ Departamento de Matemática, Universidade de Brasília, 70910-900, Brasília-DF, Brazil \\ E-mail: j.p.santos@mat.unb.br,k.tenenblat@mat.unb.br
}

Received October 01, 2012, in final form April 12, 2013; Published online April 17, 2013

http://dx.doi.org/10.3842/SIGMA.2013.033

\begin{abstract}
We consider conformally flat hypersurfaces in four dimensional space forms with their associated Guichard nets and Lamé's system of equations. We show that the symmetry group of the Lamé's system, satisfying Guichard condition, is given by translations and dilations in the independent variables and dilations in the dependents variables. We obtain the solutions which are invariant under the action of the 2-dimensional subgroups of the symmetry group. For the solutions which are invariant under translations, we obtain the corresponding conformally flat hypersurfaces and we describe the corresponding Guichard nets. We show that the coordinate surfaces of the Guichard nets have constant Gaussian curvature, and the sum of the three curvatures is equal to zero. Moreover, the Guichard nets are foliated by flat surfaces with constant mean curvature. We prove that there are solutions of the Lamé's system, given in terms of Jacobi elliptic functions, which are invariant under translations, that correspond to a new class of conformally flat hypersurfaces.
\end{abstract}

Key words: conformally flat hypersurfaces; symmetry group; Lamé's system; Guichard nets

2010 Mathematics Subject Classification: 53A35; 53C42

\section{Introduction}

The investigation of conformally flat hypersurfaces has been of interest for quite some time. Any surface in $\mathbb{R}^{3}$ is conformally flat, since it can be parametrized by isothermal coordinates. For higher dimensional hypersurfaces, E. Cartan [2] gave a complete classification for the conformally flat hypersurfaces of an $(n+1)$-dimensional space form when $n+1 \geq 5$. He proved that such hypersurfaces are quasi-umbilic, i.e., one of the principal curvatures has multiplicity at least $n-1$. In the same paper, Cartan investigated the case $n+1=4$. He showed that the quasi-umbilic surfaces are conformally flat, but the converse does not hold (for a proof see [13]). Moreover, he gave a characterization of the conformally flat 3-dimensional hypersurfaces, with three distinct principal curvatures, in terms of certain integrable distributions. Since then, there has been an effort to obtain a classification of hypersurfaces satisfying Cartan's characterization.

Lafontaine [13] considered hypersurfaces of type $M^{3}=M^{2} \times I \subset \mathbb{R}^{4}$. He obtained the following classes of conformally flat hypersurfaces: a) $M^{3}$ is a cylinder over a surface, $M^{2} \subset \mathbb{R}^{3}$, with constant curvature; b) $M^{3}$ is a cone over a surface in the sphere, $M^{2} \subset \mathbb{S}^{3}$, with constant curvature; c) $M^{3}$ is obtained by rotating a constant curvature surface of the hyperbolic space, $M^{2} \subset \mathbb{H}^{3} \subset \mathbb{R}^{4}$, where $\mathbb{H}^{3}$ is the half space model.

Motivated by Cartan's paper, Hertrich-Jeromin [8], established a correspondence between conformally flat three-dimensional hypersurfaces, with three distinct principal curvatures, and

\footnotetext{
* This paper is a contribution to the Special Issue "Symmetries of Differential Equations: Frames, Invariants and Applications". The full collection is available at http://www.emis.de/journals/SIGMA/SDE2012.html
} 
Guichard nets. These are systems of triply orthogonal surfaces originally considered by C. Guichard in [6], where he referred to those systems as the analogues of isothermal coordinates.

In view of Hertrich-Jeromin results, the problem of classifying conformally flat 3-dimensional hypersurfaces was transferred to the problem of classifying Guichard nets in $\mathbb{R}^{3}$. These are open sets of $\mathbb{R}^{3}$, with an orthogonal flat metric $g=\sum_{i=1}^{3} l_{i}^{2} d x_{i}^{2}$, where the functions $l_{i}$ satisfy the Guichard condition, namely,

$$
l_{1}^{2}-l_{2}^{2}+l_{3}^{2}=0,
$$

and a system of second-order partial differential equations, which is called Lamé's system (see $(2.2))$.

Hertrich-Jeromin obtained an example of a Guichard net, starting from surfaces parallel to Dini's helix and he proved that the corresponding conformally flat hypersurface was a new example, since it did not belong to the class described by Lafontaine.

In [20, 21, 22], Suyama extended the previous results by showing that the Guichard nets described by Hertrich-Jeromin are characterized in terms of a differentiable function $\varphi\left(x_{1}, x_{2}, x_{3}\right)$ that determines, up to conformal equivalence, the first and second fundamental forms of the corresponding conformally flat hypersurfaces. Moreover, Suyama showed that if $\varphi$ does not depend on one of the variables, then the hypersurface is conformal to one of the classes described by Lafontaine. He also showed that the function associated to the example given by HertrichJeromin satisfied $\varphi_{, x_{1} x_{2}}=\varphi_{, x_{2} x_{3}}=0$. Starting with this condition on $\varphi$, Suyama obtained a partial classification of such conformally flat hypersurfaces. The complete classification of conformally flat hypersurfaces, satisfying the above condition on the partial derivatives of $\varphi$, was obtained by Hertrich-Jeromin and Suyama in [10]. They showed that these hypersurfaces correspond to a special type of Guichard nets. The authors called them cyclic Guichard nets, due to the fact that one of the coordinates curves is contained in a circle.

In this paper, we obtain solutions $l_{i}$ satisfying Lamé's system and the Guichard condition, which are invariant under the action of the 2-dimensional subgroups of the symmetry group of the system. Moreover, we investigate the properties of the Guichard nets and of the conformally flat hypersurfaces associated to the solutions $l_{i}$. We first determine the symmetry group of Lamé's system satisfying the Guichard condition. We prove that the group is given by translations and dilations of the independent variables $x_{i}$ and dilations of the dependent variables $l_{i}$.

We obtain the solutions $l_{i}, i=1,2,3$, which are invariant under the action of the 2-dimensional translation subgroup, i.e., $l_{i}(\xi)$, where $\xi=\sum_{i=1}^{3} \alpha_{i} x_{i}$. These solutions are given explicitly in Theorem 3 by Jacobi elliptic functions, whenever all the functions $l_{i}$ are not constant and in Theorem 4 when one of the functions $l_{i}$ is constant. Moreover, we consider the solutions $l_{i}$ which are invariant under the 2-dimensional subgroup involving translations and dilations, i.e., $l_{i}(\eta)$, where $\eta=\sum_{j=1}^{3} a_{j} x_{j} / \sum_{k=1}^{3} b_{k} x_{k}$. In this case, if we require the functions $l_{i}(\eta)$ to depend on all three variables, then $l_{i}$ are constant functions. Otherwise, the solutions $l_{i}(\eta)$ are given explicitly in Theorem 5. The symmetry subgroup of dilations on the dependent variables is irrelevant for the study of conformally flat hypersurfaces.

Considering the functions $l_{i}$ which are invariant under the action of translations, we study the corresponding Guichard nets. We show that their coordinate surfaces have constant Gaussian curvature and the sum of the three curvatures is equal to zero. Moreover the Guichard nets are foliated by flat surfaces, with constant mean curvature.

Finally, we investigate the conformally flat hypersurfaces associated to the functions $l_{i}$ which are invariant under the action of translations. We show that, whenever the basic invariant $\xi$ depends on two variables, the hypersurface is conformal to one of the products considered by 
Lafontaine. In this case, the three-dimensional conformally flat hypersurfaces are constructed from flat surfaces contained in the hyperbolic 3 -space $\mathbb{H}^{3}$ or in the sphere $\mathbb{S}^{3}$. Whenever the basic invariant $\xi$ depends on all three independent variables, then the functions $l_{i}(\xi)$, which are given in terms of Jacobi elliptic functions, produce a new class of conformally flat hypersurfaces.

In Section 2, we review the correspondence between conformally flat 3-dimensional hypersurfaces with Lamé's system, and Guichard nets.

In Section 3, we obtain the symmetry group of Lamé's system satisfying Guichard condition and the solutions which are invariant under 2-dimensional subgroups of the symmetry group. The motivation and the technique used in this section were inspired by the fact that our system of differential equations is quite similar to the intrinsic generalized wave and sine-Gordon equations and the generalized Laplace and sinh-Gordon equations. The symmetry groups of these systems and the solutions invariant under subgroups were obtained by Tenenblat and Winternitz in [24] and Ferreira [4]. The geometric properties of the submanifolds corresponding to the solutions invariant under the subgroups of symmetries can be found in [1] and [19].

In Sections 4 and 5, we describe the geometric properties of the Guichard nets and of the conformally flat hypersurfaces that are associated to the solutions of Lamé's system which are invariant under the action of the translation group.

The solutions $l_{i}$ of Lamé's system, satisfying Guichard condition, which are invariant under the subgroup of dilations of the independent variables and the corresponding geometric theory, will be considered in another paper. Such solutions are obtained by solving a (reduced) system of partial differential equations, in contrast to what occurs in this paper, where the Lamé's system is reduced to a system of ordinary differential equations.

\section{Lamé's system and conformally flat hypersurfaces}

Consider the Minkowski space $\mathbb{R}_{1}^{6}$ with coordinates $\left(x_{0}, \ldots, x_{5}\right)$ and the scalar product $\langle$, given by

$$
\begin{aligned}
\langle,\rangle: & \mathbb{R}^{6} \times \mathbb{R}^{6} \longrightarrow \mathbb{R}, \\
(v, w) & \mapsto-v_{0} w_{0}+\sum_{i=1}^{5} v_{i} w_{i} .
\end{aligned}
$$

Let $L^{5}=\left\{y \in \mathbb{R}_{1}^{6} \mid\langle y, y\rangle=0\right\}$, be the light cone in $\mathbb{R}_{1}^{6}$ and consider $m_{K} \in \mathbb{R}_{1}^{6}$, with $\left\langle m_{K}, m_{K}\right\rangle=K$. Then, it is not difficult to see that, the sets

$$
M_{K}^{4}=\left\{y \in L^{5} \mid\left\langle y, m_{K}\right\rangle=-1\right\},
$$

with the metric induced from $\mathbb{R}_{1}^{6}$, are complete Riemannian manifolds with constant sectional curvature $K$. If $K<0$, then $M_{K}^{4}$ consists of two connected components which can be isometrically identified (see [7, Lemma 1.4.1] for details).

With this approach, consider a Riemannian immersion $f: M^{3} \rightarrow M_{K}^{4} \subset L^{5}$, with unit normal $n$. Then $\langle d f, n\rangle \equiv 0$, and $n$ also satisfies $\left\langle n, m_{K}\right\rangle=\langle n, f\rangle=0$. Let $\tilde{f}: M^{3} \rightarrow L^{5}$ be an immersion given by $\tilde{f}=e^{u} f$, where $u$ is a differentiable function on $M$. Observe that the metric induced on $\tilde{f}$ is conformal to the metric induced on the immersion $f$, i.e.,

$$
\langle d \tilde{f}, d \tilde{f}\rangle=e^{2 u}\langle d f, d f\rangle .
$$

Definition 1. Let $f: M^{3} \rightarrow L^{5}$ be an immersion such that the induced metric, $\langle d f, d f\rangle$, is positive definite. Let $n$ be a unit normal with $\langle f, n\rangle=0$ and consider differentiable functions $u$ and $a$ on $M^{3}$. Then the pair $(f, n)$ is called a strip and the pair $(\tilde{f}, \tilde{n})$ given by

$$
\tilde{f}=e^{u} f, \quad \tilde{n}=n+a f
$$

is called a conformal deformation of the strip $(f, n)$. 
Therefore, we can deform a conformally flat immersion in a space form $f: M^{3} \rightarrow M_{K}^{4} \subset L^{5}$ to a flat immersion in the light cone $\tilde{f}: M^{3} \rightarrow L^{5}$, by considering a conformal deformation, and vice-versa. Hence the problem of investigating conformally flat hypersurfaces in space forms reduces to a problem of studying flat immersions in the light cone $f: M^{3} \rightarrow L^{5}$. We say that a conformally flat hypersurface in a space form $M_{k}^{4}$ is generic if it has three distinct principal curvatures. Hertrich-Jeromin in [8] established a relation between generic conformally flat hypersurfaces in $M_{k}^{4}$ and Guichard nets [6]. Namely, let $e_{1}, e_{2}, e_{3}$ be an orthonormal frame tangent to $M^{3} \subset M_{k}^{4}$, such that $e_{i}$ are principal directions. Let $\omega_{1}, \omega_{2}, \omega_{3}$ be the co-frame and let $k_{1}, k_{2}, k_{3}$ be the principal curvatures. Assume that locally $k_{3}>k_{2}>k_{1}$, then the conformal fundamental forms

$$
\begin{aligned}
& \alpha_{1}=\sqrt{\left(k_{3}-k_{1}\right)\left(k_{2}-k_{1}\right)} \omega_{1}, \quad \alpha_{2}=\sqrt{\left(k_{2}-k_{1}\right)\left(k_{3}-k_{2}\right)} \omega_{2}, \\
& \alpha_{3}=\sqrt{\left(k_{3}-k_{2}\right)\left(k_{3}-k_{1}\right)} \omega_{3}
\end{aligned}
$$

are closed, if and only if, the hypersurface $M^{3}$ is conformally flat. Therefore, when $\alpha_{i}$ are closed forms, locally there exist $x_{1}, x_{2}, x_{3}$ such that $\alpha_{1}=d x_{1}, \alpha_{2}=d x_{2}$ and $\alpha_{3}=d x_{3}$. By integration, we obtain a special principal coordinate system $x_{1}, x_{2}, x_{3}$ for a conformally flat hypersurface in $M_{K}^{4}$.

Definition 2. A triply orthogonal coordinate system in a Riemannian 3-manifold $(M, g)$

$$
x=\left(x_{1}, x_{2}, x_{3}\right):(M, g) \rightarrow \mathbb{R}^{3},
$$

where the functions $l_{i}=\sqrt{g\left(\partial_{x_{i}}, \partial_{x_{i}}\right)}$ satisfy the Guichard condition

$$
l_{1}^{2}-l_{2}^{2}+l_{3}^{2}=0,
$$

is called a Guichard net.

Since we can deform a conformally flat immersion in a space form into a flat immersion in the light cone, we can consider Guichard nets for flat immersions $f: M^{3} \rightarrow L^{5}$. For such a flat immersion, we express the induced metric $g=\langle d f, d f\rangle$, in terms of the Guichard net, as

$$
g=l_{1}^{2} d x_{1}^{2}+l_{2}^{2} d x_{2}^{2}+l_{3}^{2} d x_{3}^{2} .
$$

Since the metric is flat, the functions $l_{i}$ must satisfy the Lamé's system [14, pp. 73-78]:

$$
\begin{aligned}
& \frac{\partial^{2} l_{i}}{\partial x_{j} \partial x_{k}}-\frac{1}{l_{j}} \frac{\partial l_{i}}{\partial x_{j}} \frac{\partial l_{j}}{\partial x_{k}}-\frac{1}{l_{k}} \frac{\partial l_{i}}{\partial x_{k}} \frac{\partial l_{k}}{\partial x_{j}}=0, \\
& \frac{\partial}{\partial x_{j}}\left(\frac{1}{l_{j}} \frac{\partial l_{i}}{\partial x_{j}}\right)+\frac{\partial}{\partial x_{i}}\left(\frac{1}{l_{i}} \frac{\partial l_{j}}{\partial x_{i}}\right)+\frac{1}{l_{k}^{2}} \frac{\partial l_{i}}{\partial x_{k}} \frac{\partial l_{j}}{\partial x_{k}}=0 .
\end{aligned}
$$

for $i, j, k$ distinct. Moreover, if $f: M^{3} \rightarrow L^{5}$ is flat, we can consider $M^{3}$ as a subset of the Euclidean space $\mathbb{R}^{3}$ and $f$ as an isometric immersion. Then we have a Guichard net on an open subset of $\mathbb{R}^{3}$, by considering as in Definition 2, $x: U \subset \mathbb{R}^{3} \rightarrow \mathbb{R}^{3}$, where the functions $l_{i}$ satisfy the Guichard condition (2.1) and the Lamé's system (2.2). At this point, one can ask if such a Guichard net determines a conformally flat hypersurface in a space form, or equivalently, a flat immersion in $L^{5}$. The answer to this question was given by the following fundamental result due to Hertrich-Jeromin [8]:

Theorem 1. For any generic conformally flat hypersurface of a space form $M_{K}^{4}$, there exists a Guichard net $x: U \subset \mathbb{R}^{3} \rightarrow \mathbb{R}^{3}$ on an open set $U$ of the Euclidean space $\mathbb{R}^{3}$ (uniquely determined up to a Möbius transformation of $\mathbb{R}^{3}$ ). 
Conversely, given a Guichard net $x=\left(x_{1}, x_{2}, x_{3}\right): U \subset \mathbb{R}^{3} \rightarrow \mathbb{R}^{3}$ for the Euclidean space, with $l_{i}=\sqrt{g\left(\partial_{x_{i}}, \partial_{x_{j}}\right)}$, where $g$ is the canonical flat metric, there exists a generic conformally flat hypersurface in a space form $M_{K}^{4}$ (in this case, Möbius equivalent Guichard nets are related to conformally equivalent immersions), whose induced metric is given by

$$
g=e^{2 P(x)}\left\{l_{1}^{2} d x_{1}^{2}+l_{2}^{2} d x_{2}^{2}+l_{3}^{2} d x_{3}^{2}\right\},
$$

where $P(x)$ is a function depending on $M_{K}^{4}$.

The converse is based on the fact that the functions $l_{i}$ determine the connection forms of a flat immersion $f: M^{3} \rightarrow L^{5}$. In fact, these connection forms satisfy the Maurer-Cartan equations if, and only if, the functions $l_{i}$ satisfy the Guichard condition and the Lamé's system.

Therefore, one way of obtaining generic conformally flat hypersurfaces in space forms $M_{K}^{4}$ is finding solutions of Lamé's system, satisfying the Guichard condition. Then the hypersurfaces are constructed by using Theorem 1. Our objective is to obtain a class of such solutions and to investigate the associated Guichard nets as well as the conformally flat hypersurfaces. We will use the theory of Lie point symmetry groups of differential equations, to obtain the symmetry group of Lamé's system and their solutions invariant under the action of subgroups of the symmetry group. This is the content of the following sections.

\section{The symmetry group of Lamé's system}

In this section, we obtain the Lie point symmetry group of Lamé's system. We start with a brief introduction of symmetry groups of differential equations. The reader who is familiar to the theory may skip this introduction.

The theory of Lie point symmetry group is an important tool for the analysis of differential equations developed by Lie at the end of the nineteen century [15]. Roughly speaking, Lie point symmetries of a system of differential equations consist of a Lie group of transformations acting on the dependent and independent variables, that transform solutions of the system into solutions.

A standard reference for the theory of symmetry groups of differential equations is Olver's book [17], where a clear approach to the subject is given, with theoretical foundations and a large number of examples and techniques. We will describe here some basic concepts that will be used in this section.

A system $S$ of $n$-th order differential equations in $p$ independent and $q$ dependent variables is given as a system of equations

$$
\Delta_{r}\left(x, u^{(n)}\right)=0, \quad v=1, \ldots, l,
$$

involving $x=\left(x_{1}, \ldots, x_{p}\right), u=\left(u_{1}, \ldots, u_{q}\right)$ and the derivatives $u^{(n)}$ of $u$ with respect to $x$ up to order $n$.

A symmetry group of the system $S$ is a local Lie group of transformations $G$ acting on an open subset $M \subset X \times U$ of the space of independent and dependent variables for the system, with the property that whenever $u=f(x)$ is a solution of $S$, and whenever $g f$ is defined for $g \in G$, then $u=g f(x)$ is also a solution of the system. A vector field $\mathbf{v}$ in the Lie algebra $\mathfrak{g}$ of the group $G$ is called an infinitesimal generator.

Consider $\mathbf{v}$ as a vector field on $M \subset X \times U$, with corresponding (local) one-parameter group $\exp (\varepsilon \mathbf{v})$, i.e.,

$$
\exp (\varepsilon \mathbf{v}) \equiv \Psi(\varepsilon, x)
$$


where $\Psi$ is the flow generated by $\mathbf{v}$. In this case, $\mathbf{v}$ will be the infinitesimal generator of the action.

The symmetry group of a given system of differential equation, is obtained by using the prolongation formula and the infinitesimal criterion that are described as follows. Given a vector field on $M \subset X \times U$,

$$
\mathbf{v}=\sum_{i=1}^{p} \xi^{i}(x, u) \frac{\partial}{\partial x_{i}}+\sum_{\alpha=1}^{q} \phi_{\alpha}(x, u) \frac{\partial}{\partial u^{\alpha}},
$$

the $n$-th prolongation of $\mathbf{v}$ is the vector field

$$
\operatorname{pr}^{(n)} \mathbf{v}=\mathbf{v}+\sum_{\alpha=1}^{q} \sum_{J} \phi_{\alpha}^{J}\left(x, u^{(n)}\right) \frac{\partial}{\partial u_{J}^{\alpha}} .
$$

It is defined on the corresponding jet space $M^{(n)} \subset X \times U^{(n)}$, whose coordinates represent the independent variables, the dependent variables and the derivatives of the dependent variables up to order $n$. The second summation is taken over all (unordered) multi-indices $J=\left(j_{1}, \ldots, j_{k}\right)$, with $1 \leq j_{k} \leq p, 1 \leq k \leq n$. The coefficient functions $\phi_{\alpha}^{J}$ of $\operatorname{pr}^{(n)} \mathbf{v}$ are given by the following formula:

$$
\phi_{\alpha}^{J}\left(x, u^{(n)}\right)=D_{J}\left(\phi_{\alpha}-\sum_{i=1}^{p} \xi^{i} u_{J, i}^{\alpha},\right),
$$

where $u_{i}^{\alpha}=\frac{\partial u^{\alpha}}{\partial x_{i}}, u_{J, i}^{\alpha}=\frac{\partial u_{J}^{\alpha}}{\partial x_{i}}$ and $D_{J}$ is given by the total derivatives

$$
D_{J}=D_{j_{1}} D_{j_{2}} \cdots D_{j_{k}},
$$

with

$$
D_{i} f\left(x, u^{(n)}\right)=\frac{\partial f}{\partial x_{i}}+\sum_{\alpha_{1}}^{p} \sum_{J} u_{J, i}^{\alpha} \frac{\partial f}{\partial u_{J}^{\alpha}} .
$$

We say that the system (3.1) is a system of maximal rank over $M \subset X \times U$, if the Jacobian matrix

$$
J_{\Delta}\left(x, u^{(n)}\right)=\left(\frac{\partial \Delta_{r}}{\partial x_{i}}, \frac{\partial \Delta_{r}}{\partial u_{, \mathbf{J}}^{\alpha}}\right)
$$

has rank $l$, whenever $\Delta_{r}\left(x, u^{(n)}\right)=0$, where $\mathbf{J}=\left(j_{1}, \ldots, j_{k}\right)$ is a multi-index that denotes the partial derivatives of $u^{\alpha}$.

Suppose that (3.1) is a system of maximal rank. Then the set of all vectors fields $\mathbf{v}$ on $M$ such that

$$
\operatorname{pr}^{(n)} \mathbf{v}\left[\Delta_{r}\left(x, u^{(n)}\right)\right]=0, \quad r=1, \ldots, l, \quad \text { whenever } \quad \Delta_{r}\left(x, u^{(n)}\right)=0,
$$

is a Lie algebra of infinitesimal generators of a symmetry group for the system. It is shown in [17] that the infinitesimal criterion (3.2) is in fact both a necessary and sufficient condition for a group $G$ to be a symmetry group. Hence, all the connected symmetry groups can be determined by considering this criterion.

Since the prolongation formula is given in terms of $\xi^{i}$ and $\phi_{\alpha}$ and the partial derivatives with respect to both $x$ and $u$, the infinitesimal criterion provides a system of partial differential equations for the coefficients $\xi^{i}$ and $\phi_{\alpha}$ of $\mathbf{v}$, called the determining equations. By solving these equations, we obtain the vector field $\mathbf{v}$ that determines a Lie algebra $\mathfrak{g}$. The symmetry group $G$ is obtained by exponentiating the Lie algebra. 


\subsection{Obtaining the symmetry group of Lamé's system}

From now on, we consider the following notation for derivatives of a function $f=f\left(x_{1}, \ldots, x_{n}\right)$

$$
f_{, x_{i}}:=\frac{\partial f}{\partial x_{i}} \quad \text { and } \quad f_{, x_{i} x_{j}}:=\frac{\partial^{2} f}{\partial x_{i} \partial x_{j}} .
$$

With this notation, Lamé's system (2.2) is given by

$$
\begin{aligned}
& l_{i, x_{j} x_{k}}-\frac{l_{i, x_{j}} l_{j, x_{k}}}{l_{j}}-\frac{l_{i, x_{k}} l_{k, x_{j}}}{l_{k}}=0, \\
& \left(\frac{l_{i, x_{j}}}{l_{j}}\right)_{, x_{j}}+\left(\frac{l_{j, x_{i}}}{l_{i}}\right)_{, x_{i}}+\frac{l_{i, x_{k}} l_{j, x_{k}}}{l_{k}^{2}}=0,
\end{aligned}
$$

where $i, j$ and $k$ are distinct indices in the set $\{1,2,3\}$. We will also consider the following notation,

$$
\varepsilon_{s}=\left\{\begin{aligned}
1 & \text { if } s=1 \text { or } s=3 \\
-1 & \text { if } s=2
\end{aligned}\right.
$$

We can now rewrite Guichard condition as

$$
\varepsilon_{i} l_{i}^{2}+\varepsilon_{j} l_{j}^{2}+\varepsilon_{k} l_{k}^{2}=0 .
$$

Next, we introduce auxiliary functions in order to reduce the system of second-order differential equations (3.3) and (3.4), into a first order one. Consider the functions $h_{i j}$, with $i \neq j$, given by

$$
l_{i, x_{j}}-h_{i j} l_{j}=0 .
$$

With these functions, we rewrite (3.3) and (3.4) as

$$
h_{i j, x_{k}}-h_{i k} h_{k j}=0, \quad h_{i j, x_{j}}+h_{j i, x_{i}}+h_{i k} h_{j k}=0 .
$$

for $i, j, k$ distinct. Since the functions $l_{1}, l_{2}$ and $l_{3}$ satisfy Guichard condition, there are other relations involving the derivatives of $l_{i}$ and $h_{i j}$. Taking the derivative of Guichard condition with respect to $x_{i}$, we have

$$
\varepsilon_{i} l_{i, x_{i}}+\varepsilon_{j} h_{j i} l_{j}+\varepsilon_{k} h_{k i} l_{k}=0,
$$

for $i, j, k$ distinct. The derivatives of the above equation with respect to $x_{j}$ leads to

$$
\varepsilon_{i} h_{i j, x_{i}}+\varepsilon_{j} h_{j i, x_{j}}+\varepsilon_{k} h_{k i} h_{k j}=0 .
$$

Therefore, we summarize the last six equations in the following system of first-order partial differential equations, equivalent to Lamé's system, that we call Lamé's system of first order

$$
\begin{aligned}
& \varepsilon_{i} l_{i}^{2}+\varepsilon_{j} l_{j}^{2}+\varepsilon_{k} l_{k}^{2}=0, \\
& l_{i, x_{j}}-h_{i j} l_{j}=0, \\
& \varepsilon_{i} l_{i, x_{i}}+\varepsilon_{j} h_{j i} l_{j}+\varepsilon_{k} h_{k i} l_{k}=0, \\
& h_{i j, x_{k}}-h_{i k} h_{k j}=0, \\
& h_{i j, x_{j}}+h_{j i, x_{i}}+h_{i k} h_{j k}=0, \\
& \varepsilon_{i} h_{i j, x_{i}}+\varepsilon_{j} h_{j i, x_{j}}+\varepsilon_{k} h_{k i} h_{k j}=0 .
\end{aligned}
$$

By considering $x=\left(x_{1}, x_{2}, x_{3}\right), l=\left(l_{1}, l_{2}, l_{3}\right)$ and $h$ the off-diagonal $3 \times 3$ matrix given by $h_{i j}$ in our next two results, we obtain the Lie algebra of the infinitesimal generators and the symmetry group of Lamé's system of first order. 
Theorem 2. Let $V$ be the infinitesimal generator of the symmetry group of Lamé's system of first order (3.6)-(3.11), given by

$$
V=\sum_{i=1}^{3} \xi^{i}(x, l, h) \frac{\partial}{\partial x_{i}}+\sum_{i=1}^{3} \eta^{i}(x, l, h) \frac{\partial}{\partial l_{i}}+\sum_{i, j=1, i \neq j}^{3} \phi^{i j}(x, l, h) \frac{\partial}{\partial h_{i j}} .
$$

Then the functions $\xi^{i}, \eta^{i}$ and $\phi^{i j}$ are given by

$$
\xi^{i}=a x_{i}+a_{i}, \quad \eta^{i}=c l_{i}, \quad \phi^{i j}=-a h_{i j},
$$

where $a, c, a_{i} \in \mathbb{R}$.

The proof of Theorem 2 is very long and technical. It consists of obtaining the functions $\xi^{i}, \eta^{i}$ and $\phi^{i j}$ by solving the determining equations which are obtained as follows. We apply the first prolongation of $V$ to each equation (3.6)-(3.11) and we eliminate the functional dependence of the derivatives of $h$ and $l$ caused by the system. Then we equate to zero the coefficients of the remaining unconstrained partial derivatives. The complete proof with, all the details, is given in Appendix A.

As a consequence of Theorem 2, by exponentiating $V$, we obtain the symmetry group of Lamé's system. Observe that the functions $\phi^{i j}$ do not depend on $x$ and $l$ (see [18] for symmetry group of equivalent systems):

Corollary 1. The symmetry group of Lamé's system (3.6)-(3.11) is given by the following transformations:

1) translations in the independent variables: $\tilde{x}_{i}=x_{i}+v_{i}$;

2) dilations in the independent variables: $\tilde{x}_{i}=\lambda x_{i}$;

3) dilations in the dependent variables: $\tilde{l}_{i}=\rho l_{i}$;

where $v_{i} \in \mathbb{R}$ and $\lambda, \rho \in \mathbb{R} \backslash\{0\}$.

\subsection{Group invariant solutions}

The knowledge of all the infinitesimal generators $\mathbf{v}$ of the symmetry group of a system of differential equations, allows one to reduce the system to another one with a reduced number of variables. Specifically, if the system has $p$ independent variables and an $s$-dimensional symmetry subgroup is considered, then the reduced system for the solutions invariant under this subgroup will depend on $p-s$ variables (see Olver [17] for details). Finding all the $s$-dimensional symmetry subgroups is equivalent to finding all the $s$-dimensional subalgebras of the Lie algebra of infinitesimal symmetries $\mathbf{v}$. For the remainder of this paper, we will consider the 2-dimensional subgroups of the symmetry group of Lamé's system. The first one will be the translation subgroup and the second one will be the subgroup involving translations and the dilations. The 1-dimensional subgroup given just by dilations and the solutions invariant under this subgroup are being investigated. We will report on our investigation in another paper. We observe that the symmetry subgroup of dilations in the dependent variables (Corollary 1(3)) is irrelevant for the geometric study of conformally flat hypersurfaces due to (2.3).

We start with the 2-dimensional subgroup of translations. The basic invariant of this group is given by

$$
\xi=\alpha_{1} x_{1}+\alpha_{2} x_{2}+\alpha_{3} x_{3},
$$

where $\left(\alpha_{1}, \alpha_{2}, \alpha_{3}\right)$ is a non zero vector. We will consider solutions $l_{i}$ such that

$$
l_{i}\left(x_{1}, x_{2}, x_{3}\right)=l_{i}(\xi), \quad 1 \leq i \leq 3,
$$


where $\xi$ is given by (3.13). For such solutions, Lamé's system reduces to a system of ODEs. We start with two lemmas:

Lemma 1. Let $l_{s}(\xi), s=1,2,3$, where $\xi=\sum_{s=1}^{3} \alpha_{s} x_{s}$, be a solution of Lamé's system (3.6)(3.11). Let $i, k \in\{1,2,3\}$ be two fixed and distinct indices such that $\alpha_{i}=\alpha_{k}=0$. Then $l_{i}$ or $l_{k}$ is constant.

Proof. Since $\alpha_{i}=\alpha_{k}=0$, it follows from (3.7) that equation (3.10) reduces to

$$
\alpha_{j}^{2}\left[\frac{l_{i, \xi}}{l_{j}}\right]_{, \xi}=0,
$$

which implies $l_{i, \xi}=c_{i} l_{j}$, where $c_{i} \in \mathbb{R}$. Similarly, interchanging $i$ with $k$, we obtain $l_{k, \xi}=c_{k} l_{j}$. Finally, interchanging $k$ with $j$, we get

$$
\alpha_{j}^{2} \frac{l_{i, \xi} l_{k, \xi}}{l_{j}^{2}}=\alpha_{j}^{2} c_{i} c_{k}=0 .
$$

Therefore, we conclude that $l_{i}$ or $l_{k}$ is constant.

Lemma 2. Let $l_{s}(\xi), s=1,2,3$, where $\xi=\sum_{s=1}^{3} \alpha_{s} x_{s}$, be a solution of Lamé's system (3.6)-(3.11). If there exists a unique $j \in\{1,2,3\}$ such that $l_{j}$ is a non zero constant, then $\alpha_{j}=0$.

Proof. Interchanging the indices in (3.9), we obtain the following two equations

$$
\begin{aligned}
& \alpha_{j} \alpha_{k}\left(l_{i, \xi \xi}-\frac{l_{i, \xi} l_{k, \xi}}{l_{k}}\right)=0, \\
& \alpha_{j} \alpha_{i}\left(l_{k, \xi \xi}-\frac{l_{k, \xi} l_{i, \xi}}{l_{i}}\right)=0,
\end{aligned}
$$

and an identity.

Similarly, it follows from (3.10) that

$$
\begin{aligned}
& \alpha_{j}^{2} l_{i, \xi \xi}=0, \\
& \alpha_{j}^{2} l_{k, \xi \xi}=0, \\
& \alpha_{k}^{2}\left(\frac{l_{i, \xi}}{l_{k}}\right)_{, \xi}+\alpha_{i}^{2}\left(\frac{l_{k, \xi}}{l_{i}}\right)_{, \xi}+\alpha_{j}^{2} \frac{l_{i, \xi} l_{k, \xi}}{l_{j}}=0 .
\end{aligned}
$$

Suppose, by contradiction, that $\alpha_{j} \neq 0$. It follows from (3.16) and (3.17) that $l_{i, \xi}=c_{i}$ and $l_{k, \xi}=c_{k}$, where $c_{i} \neq 0$ and $c_{k} \neq 0$, since by hypothesis, $l_{i}$ and $l_{k}$ are non constants. Then, it follows from (3.14) and (3.15) that $\alpha_{i}=\alpha_{k}=0$. From (3.18), we obtain $\alpha_{j}^{2} c_{i} c_{k}=0$, which is a contradiction.

The following theorem gives the solutions of Lamé's system, satisfying Guichard condition, which are invariant under the action of the translation group, whenever none of the functions $l_{i}$ is constant.

Theorem 3. Let $l_{s}(\xi), s=1,2,3$, where $\xi=\sum_{s=1}^{3} \alpha_{s} x_{s}$, be a solution of Lamé's system (3.6)(3.11), such that $l_{s}$ is not constant for all s. Then there exist $c_{s} \in \mathbb{R} \backslash\{0\}$, such that,

$$
l_{i, \xi}=c_{i} l_{k} l_{j}, \quad i, j, k \text { distinct }
$$




$$
\begin{aligned}
& c_{1}-c_{2}+c_{3}=0, \\
& \alpha_{1}^{2} c_{2} c_{3}+\alpha_{2}^{2} c_{1} c_{3}+\alpha_{3}^{2} c_{1} c_{2}=0 .
\end{aligned}
$$

Moreover, the functions $l_{i}(\xi)$ are given by

$$
\begin{aligned}
& l_{1, \xi}^{2}=c_{2}\left(c_{2}-c_{1}\right)\left(l_{1}^{2}-\frac{\lambda}{c_{2}}\right)\left(l_{1}^{2}-\frac{\lambda}{c_{2}-c_{1}}\right), \\
& l_{2}^{2}=\frac{c_{2}}{c_{1}}\left(l_{1}^{2}-\frac{\lambda}{c_{2}}\right) \\
& l_{3}^{2}=\frac{c_{2}-c_{1}}{c_{1}}\left(l_{1}^{2}-\frac{\lambda}{c_{2}-c_{1}}\right)
\end{aligned}
$$

where $\lambda \in \mathbb{R}$.

Proof. By hypothesis, we are considering non constant solutions. Then, it follows from Lemma 1, that $\alpha_{s} \neq 0$ for at least two distinct indices. Suppose that $\alpha_{j}$ and $\alpha_{k}$ non zero. From (3.7) and (3.9) we obtain

$$
\alpha_{j} \alpha_{k}\left\{\left[\frac{l_{i, \xi}}{l_{j}}\right]_{, \xi}-\frac{l_{i, \xi}}{l_{j}} \frac{l_{k, \xi}}{l_{k}}\right\}=0
$$

which implies

$$
\left[\frac{l_{i, \xi}}{l_{j}}\right]_{, \xi}\left[\frac{l_{i, \xi}}{l_{j}}\right]^{-1}=\frac{l_{k, \xi}}{l_{k}}
$$

Integrating this equation, we obtain $l_{i, \xi}=c_{i} l_{k} l_{j}$, where $c_{i} \neq 0$.

If $\alpha_{i} \neq 0$, analogously considering the non zero pairs $\left(\alpha_{i}, \alpha_{j}\right)$ and $\left(\alpha_{i}, \alpha_{k}\right)$, we conclude that $l_{k, \xi}=c_{k} l_{i} l_{j}$ and $l_{j, \xi}=c_{j} l_{i} l_{k}$. If $\alpha_{i}=0$, then from equation (3.10) we have

$$
\left[\frac{l_{i, x_{j}}}{l_{j}}\right]_{, x_{j}}+\frac{l_{i, x_{k}}}{l_{k}} \frac{l_{j, x_{k}}}{l_{k}}=\alpha_{j}^{2} c_{i} l_{k, \xi}+\alpha_{k}^{2} c_{i} l_{j} \frac{l_{j, \xi}}{l_{k}}=0 .
$$

Since $c_{i} \neq 0$, we integrate the above expression to obtain

$$
\alpha_{j}^{2} l_{k}^{2}+\alpha_{k}^{2} l_{j}^{2}=\lambda_{j k},
$$

where $\lambda_{j k}$ is a constant. This equation and Guichard condition (3.6) lead to

$$
l_{j}^{2}=\frac{\alpha_{j}^{2}}{\alpha_{k}^{2}}\left(\frac{\lambda_{j k}}{\alpha_{j}^{2}}-l_{k}^{2}\right), \quad l_{i}^{2}=\frac{\varepsilon_{i}}{\alpha_{k}^{2}}\left[l_{k}^{2}\left(\varepsilon_{j} \alpha_{j}^{2}-\varepsilon_{k} \alpha_{k}^{2}\right)-\varepsilon_{j} \lambda_{j k}\right] .
$$

Taking the derivative of the last equation with respect to $\xi$, we have

$$
l_{i}\left(c_{i} l_{k} l_{j}\right)=\frac{\varepsilon_{i}}{\alpha_{k}^{2}}\left[l_{k} l_{k, \xi}\left(\varepsilon_{j} \alpha_{j}^{2}-\varepsilon_{k} \alpha_{k}^{2}\right)\right] .
$$

If $\varepsilon_{j} \alpha_{j}^{2}-\varepsilon_{k} \alpha_{k}^{2} \neq 0$, we conclude that

$$
l_{k, \xi}=\frac{c_{i} \alpha_{k}^{2}}{\varepsilon_{j} \alpha_{j}^{2}-\varepsilon_{k} \alpha_{k}^{2}} l_{i} l_{j}=c_{k} l_{i} l_{j} .
$$


Applying this expression into the derivative of the first equation in (3.25) with respect to $\xi$ we obtain

$$
l_{j} l_{j, \xi}=-\frac{\alpha_{j}^{2}}{\alpha_{k}^{2}} l_{k} l_{k, \xi}=-\frac{\alpha_{j}^{2}}{\alpha_{k}^{2}} l_{k}\left(c_{k} l_{i} l_{j}\right),
$$

consequently, $l_{j, \xi}=c_{j} l_{i} l_{k}$.

Next, we will show that $\varepsilon_{j} \alpha_{j}^{2}-\varepsilon_{k} \alpha_{k}^{2} \neq 0$ to conclude the proof of (3.19). Suppose by contradiction that $\varepsilon_{j} \alpha_{j}^{2}-\varepsilon_{k} \alpha_{k}^{2}=0$, then the first equation of (3.25) can be written as $\varepsilon_{j} l_{j}^{2}+\varepsilon_{k} l_{k}^{2}=$ $\frac{\varepsilon_{k} \lambda_{j k}}{\alpha_{j}^{2}}$. Then Guichard condition now implies that $l_{i}$ is constant, which is a contradiction. The relations between the constants (3.20) and (3.21) follow from a straightforward computation using equations (3.8) and (3.10), respectively.

In order to complete the proof of the theorem, we start with

$$
\begin{aligned}
& l_{1, \xi}=c_{1} l_{2} l_{3}, \\
& l_{2, \xi}=c_{2} l_{1} l_{3}, \\
& l_{3, \xi}=c_{3} l_{1} l_{2} .
\end{aligned}
$$

Multiplying (3.27) by $l_{2}$ and integrating we have

$$
l_{2}^{2}=\frac{c_{2}}{c_{1}}\left(l_{1}^{2}-\frac{\lambda}{c_{2}}\right)
$$

where $\lambda$ is a constant. Therefore, it follows from (3.29) and Guichard condition that

$$
l_{3}^{2}=\frac{c_{2}-c_{1}}{c_{1}}\left(l_{1}^{2}-\frac{\lambda}{c_{2}-c_{1}}\right) .
$$

Using (3.26), (3.29) and (3.30), we conclude that

$$
\begin{aligned}
l_{1, \xi}^{2} & =c_{1}^{2}\left[\frac{c_{2}}{c_{1}}\left(l_{1}^{2}-\frac{\lambda}{c_{2}}\right)\right]\left[\frac{c_{2}-c_{1}}{c_{1}}\left(l_{1}^{2}-\frac{\lambda}{c_{2}-c_{1}}\right)\right] \\
& =c_{2}\left(c_{2}-c_{1}\right)\left(l_{1}^{2}-\frac{\lambda}{c_{2}}\right)\left(l_{1}^{2}-\frac{\lambda}{c_{2}-c_{1}}\right) .
\end{aligned}
$$

In our next theorem, we consider the solutions $l_{i}(\xi)$ when one of the functions $l_{i}$ is constant.

Theorem 4. Let $l_{s}(\xi), s=1,2,3$, where $\xi=\sum_{s=1}^{3} \alpha_{s} x_{s}$, be a solution of Lamés system (3.6)(3.11). Suppose that only one of the functions $l_{s}$ is constant. Then one of the following occur:

a) $l_{1}=\lambda_{1}, l_{2}=\lambda_{1} \cosh \left(b \xi+\xi_{0}\right), l_{3}=\lambda_{1} \sinh \left(b \xi+\xi_{0}\right)$, where $\xi=\alpha_{2} x_{2}+\alpha_{3} x_{3}, \alpha_{2}^{2}+\alpha_{3}^{2} \neq 0$ and $b, \xi_{0} \in \mathbb{R}$;

b) $l_{2}=\lambda_{2}, l_{1}=\lambda_{2} \cos \varphi(\xi), l_{3}=\lambda_{2} \sin \varphi(\xi)$, where $\xi=\alpha_{1} x_{1}+\alpha_{3} x_{3}, \alpha_{1}^{2}+\alpha_{3}^{2} \neq 0$ and $\varphi$ is one of the following:

b.1) $\varphi(\xi)=b \xi+\xi_{0}$, if $\alpha_{1}^{2} \neq \alpha_{3}^{2}$, where $\xi_{0}, b \in \mathbb{R}$;

b.2) $\varphi$ is any function of $\xi$, if $\alpha_{1}^{2}=\alpha_{3}^{2}$;

c) $l_{3}=\lambda_{3}, l_{2}=\lambda_{3} \cosh \left(b \xi+\xi_{0}\right), l_{1}=\lambda_{3} \sinh \left(b \xi+\xi_{0}\right)$, where $\xi=\alpha_{1} x_{1}+\alpha_{2} x_{2}, \alpha_{1}^{2}+\alpha_{2}^{2} \neq 0$ and $b, \xi_{0} \in \mathbb{R}$. 
Proof. We will consider each case separately:

a) If $l_{1}=\lambda_{1}$, then it follows from Lemma 2 that we must have $\xi=\alpha_{2} x_{2}+\alpha_{3} x_{3}$. Now Guichard condition implies that $l_{2}=\lambda_{1} \cosh \varphi(\xi)$ and $l_{3}=\lambda_{1} \sinh \varphi(\xi)$. In order to determine $\varphi$, we use (3.10) with the following indices

$$
h_{23, x_{3}}+h_{32, x_{2}}+h_{21} h_{31}=0,
$$

to obtain

$$
\alpha_{3}^{2}\left(\frac{\lambda_{1} \varphi_{, \xi} \sinh \varphi}{\lambda_{1} \sinh \varphi}\right)_{, \xi}+\alpha_{2}^{2}\left(\frac{\lambda_{1} \varphi_{, \xi} \cosh \varphi}{\lambda_{1} \cosh \varphi}\right)_{, \xi}=0 .
$$

Since $l_{2}$ and $l_{3}$ are not constant, we have $\alpha_{2}^{2}+\alpha_{3}^{2} \neq 0$, which implies $\varphi_{, \xi \xi}=0$. Consequently, $\varphi(\xi)=b \xi+\xi_{0}$.

b) If $l_{2}=\lambda_{2}$, it follows from Lemma 2 that $\xi=\alpha_{1} x_{1}+\alpha_{3} x_{3}$. Then Guichard condition implies that $l_{1}=\lambda_{2} \cos \varphi(\xi)$ and $l_{3}=\lambda_{2} \sin \varphi(\xi)$. As in the case $a$ ), from equation (3.10) we get $\left(\alpha_{1}^{2}-\alpha_{3}^{2}\right) \varphi_{, \xi \xi}=0$. Since $l_{1}$ and $l_{3}$ are non constant, we have $\alpha_{1}^{2}+\alpha_{3}^{2} \neq 0$. Then we have two cases to consider:

b.1) If $\alpha_{1}^{2} \neq \alpha_{3}^{2}$, then $\varphi(\xi)=b \xi+\xi_{0}$;

$b .2$ ) If $\alpha_{1}^{2}=\alpha_{3}^{2}$, then $\varphi$ can be any function of $\xi$.

c) The proof is the same as in $a$ ).

Next, we consider the solutions invariant under the 2-dimensional subgroup involving translations and dilations. In this case, the basic invariant is given by

$$
\eta=\frac{a_{1} x_{1}+a_{2} x_{2}+a_{3} x_{3}}{b_{1} x_{1}+b_{2} x_{2}+b_{3} x_{3}}
$$

where the vectors $\left(a_{1}, a_{2}, a_{3}\right)$ and $\left(b_{1}, b_{2}, b_{3}\right)$ are linearly independent. If $f=f(\eta)$ is a function depending on $\eta$, then

$$
f_{, x_{i}}=f_{, \eta} \eta_{x_{i}}=\frac{a_{i}-b_{i} \eta}{b_{1} x_{1}+b_{2} x_{2}+b_{3} x_{3}} f_{, \eta} .
$$

In order to simplify the computations, we will use the following notation:

$$
N_{i}:=a_{i}-b_{i} \eta \quad \text { and } \quad \beta=b_{1} x_{1}+b_{2} x_{2}+b_{3} x_{3} .
$$

Then we have $\eta_{, x_{i}}=\frac{N_{i}}{\beta}$.

In order to obtain the solutions of Lamé's system $l_{i}(\eta)$, which depend on $\eta$, we will need some lemmas.

Lemma 3. Let $l_{1}(\eta), l_{2}(\eta), l_{3}(\eta)$, where $\eta$ is given by (3.31), be a solution of Lamé's system (3.6)-(3.11). Suppose that for a fixed pair $j, k \in\{1,2,3\}, j \neq k,\left(a_{j}, b_{j}\right) \neq(0,0)$ and $\left(a_{k}, b_{k}\right) \neq$ $(0,0)$. Then there exists $c_{i} \in \mathbb{R}$ such that

$$
l_{i, \eta}=c_{i} \frac{l_{k} l_{j}}{N_{k} N_{j}}, \quad i \neq j, k,
$$

where $N_{k}$ is given by (3.32).

Proof. From (3.7), we have that $h_{i j}=\frac{l_{i, \eta} N_{j}}{l_{j} \beta}$. Then, equation (3.9) can be written as

$$
\left[\frac{l_{i, \eta} N_{k} N_{j}}{l_{j}}\right]_{\eta}-\frac{l_{i, \eta} N_{k} N_{j}}{l_{j}} \frac{l_{k, \eta}}{l_{k}}=0,
$$


which implies

$$
\left(\frac{l_{i, \eta} N_{k} N_{j}}{l_{k} l_{j}}\right)_{, \eta}=0
$$

Since $\left(a_{j}, b_{j}\right) \neq(0,0)$ and $\left(a_{k}, b_{k}\right) \neq(0,0)$, we have that $N_{j} \neq 0, N_{k} \neq 0$ and the equation (3.33) holds.

Lemma 4. Let $l_{1}(\eta), l_{2}(\eta), l_{3}(\eta)$, where $\eta$ is given by (3.31), be a solution of Lamé's system (3.6)-(3.11). If $\left(a_{i}, b_{i}\right)=(0,0)$, for some $i \in\{1,2,3\}$, then $l_{i}$ is constant.

Proof. Since the vectors $\left(a_{1}, a_{2}, a_{3}\right)$ and $\left(b_{1}, b_{2}, b_{3}\right)$ are linearly independent, if $\left(a_{i}, b_{i}\right)=(0,0)$ we must have $\left(a_{j}, b_{j}\right) \neq(0,0)$ and $\left(a_{k}, b_{k}\right) \neq(0,0)$ for $i, j, k$ distinct and we can use Lemma 3 . By considering equation (3.10), we have

$$
\left(\frac{c_{i} l_{k}}{\beta N_{k}}\right)_{, \eta}+\left(\frac{c_{i} l_{k}}{\beta N_{k}}\right) \frac{l_{j, \eta N_{k}}}{\beta l_{k}}=0
$$

which implies

$$
c_{i}\left[\frac{l_{k, \eta} N_{j}}{N_{k}}-\frac{l_{k}}{N_{k}^{2}}\left(N_{k} \beta\right)_{, x_{j}}+\frac{l_{j} l_{j, \eta} N_{k}}{l_{k} N_{j}}\right]=0 .
$$

By interchanging $j$ with $k$, we have analogously

$$
c_{i}\left[\frac{l_{j, \eta} N_{k}}{N_{j}}-\frac{l_{j}}{N_{j}^{2}}\left(N_{j} \beta\right)_{, x_{k}}+\frac{l_{k} l_{k, \eta} N_{j}}{l_{j} N_{k}}\right]=0 .
$$

Suppose by contradiction that $c_{i} \neq 0$. Then, it follows from (3.34) and (3.35) that

$$
\frac{l_{k}^{2}}{N_{k}^{2}}\left(N_{k} \beta\right)_{x_{j}}=\frac{l_{j}^{2}}{N_{j}^{2}}\left(N_{j} \beta\right)_{x_{k}} .
$$

If $a_{i}=b_{i}=0$, we must have

$$
\left(a_{k} b_{j}-b_{k} a_{j}\right)\left(\frac{l_{k}^{2}}{N_{k}^{2}}+\frac{l_{j}^{2}}{N_{j}^{2}}\right)=0,
$$

which is a contradiction since $\left(a_{k} b_{j}-b_{k} a_{j}\right) \neq 0$. Therefore $c_{i}=0$ and $l_{i}$ is constant.

Lemma 5. Let $l_{1}(\eta), l_{2}(\eta), l_{3}(\eta)$, with $\eta$ given by (3.31), be a solution of Lamé's system (3.6)(3.11). If there exists a unique function $l_{i}$ which is a non zero constant, then $\left(a_{i}, b_{i}\right)=(0,0)$.

Proof. Suppose by contradiction that $\left(a_{i}, b_{i}\right) \neq(0,0)$. Since $l_{j}$ and $l_{k}$ are not constant, for $i, j, k$ distinct, it follows from Lemma 4 , that we must have $\left(a_{j}, b_{j}\right) \neq(0,0)$ and $\left(a_{k}, b_{k}\right) \neq(0,0)$. Then, Lemma 3 implies that there are constants $c_{i}, c_{j}$ and $c_{k}$ such that

$$
l_{i, \eta}=c_{i} \frac{l_{j} l_{k}}{N_{j} N_{k}}, \quad l_{j, \eta}=c_{j} \frac{l_{k} l_{i}}{N_{k} N_{i}} \quad \text { and } \quad l_{k, \eta}=c_{k} \frac{l_{k} l_{i}}{N_{k} N_{i}} .
$$

Using equation (3.10) and interchanging the indices we have

$$
c_{k} \frac{l_{i} l_{j}}{N_{j}}-\frac{\left(a_{k} b_{i}-b_{k} a_{i}\right) l_{k}}{N_{k}}=0
$$




$$
\begin{aligned}
& c_{j} c_{k} \frac{l_{j} l_{k}}{N_{j} N_{k}}-\frac{l_{i}}{N_{i}^{2}}\left[c_{j}\left(a_{i} b_{k}-a_{k} b_{i}\right)+c_{k}\left(a_{i} b_{j}-b_{i} a_{j}\right)\right]=0, \\
& c_{j} \frac{l_{i} l_{k}}{N_{k}}-\frac{\left(a_{j} b_{i}-b_{j} a_{i}\right) l_{j}}{N_{j}}=0 .
\end{aligned}
$$

Multiplying equation (3.36) by $c_{j} \frac{N_{k}}{l_{k}},(3.37)$ by $\frac{N_{i}^{2}}{l_{i}}$ and (3.38) by $c_{k} \frac{N_{j}}{l_{j}}$, the sum will reduce to

$$
c_{j} c_{k}\left[\left(l_{i} l_{j} N_{k}\right)^{2}+\left(l_{i} l_{k} N_{j}\right)^{2}+\left(l_{j} l_{k} N_{i}\right)^{2}\right]=0,
$$

which is a contradiction. Then, we must have $\left(a_{i}, b_{i}\right)=(0,0)$ and the lemma is proved.

Remark 1. We observe that when all pairs $\left(a_{s}, b_{s}\right)$ are different from zero, then the proof of Lemma 5 shows that the solution $l_{i}(\eta)$ of Lamé's system is constant.

We will now obtain the solutions $l_{s}(\eta)$, when one pair $\left(a_{s}, b_{s}\right)=(0,0)$.

Theorem 5. Let $l_{i}(\eta)$, with $\eta$ given by (3.31), be a solution of Lamé's system invariant under the 2-dimensional subgroup involving translation and dilations. Suppose that one of the pairs $\left(a_{s}, b_{s}\right)=(0,0)$. Then one of the following occur:

a) If $\left(a_{1}, b_{1}\right)=(0,0)$ then $l_{1}=\lambda_{1}, l_{2}=\lambda_{1} \cosh \varphi(\eta), l_{3}=\lambda_{1} \sinh \varphi(\eta)$, where $\eta=\frac{a_{2} x_{2}+a_{3} x_{3}}{b_{2} x_{2}+b_{3} x_{3}}$ and $\varphi$ is given by

$$
\varphi(\eta)=\frac{C_{0}}{a_{2} b_{3}-a_{3} b_{2}} \arctan \left[\frac{b_{2}^{2}+b_{3}^{2}}{a_{3} b_{2}-a_{2} b_{3}}\left(\eta-\frac{a_{2} b_{2}+a_{3} b_{3}}{b_{2}^{2}+b_{3}^{2}}\right)\right]+C_{1},
$$

where $C_{0}, C_{1} \in \mathbb{R}$.

b) If $\left(a_{2}, b_{2}\right)=(0,0)$ then $l_{2}=\lambda_{2}, l_{1}=\lambda_{2} \cos \varphi(\eta), l_{3}=\lambda_{2} \sin \varphi(\eta)$, where $\eta=\frac{a_{1} x_{1}+a_{3} x_{3}}{b_{1} x_{1}+b_{3} x_{3}}$ and $\varphi$ is given as follows:

b.1) if $b_{1}=b_{3}=b$, then

$$
\varphi(\eta)=\frac{D_{0}}{2 b\left(a_{3}-a_{1}\right)} \log \left(2 b \eta-a_{1}-a_{3}\right)+D_{1},
$$

where $D_{0}, D_{1} \in \mathbb{R}$;

b.2) if $b_{1} \neq b_{3}$, then

$$
\varphi(\eta)=\frac{D_{2}}{2\left(a_{1} b_{3}-a_{3} b_{1}\right)} \log \left[\frac{\left(b_{3}+b_{1}\right) \eta-\left(a_{3}+a_{1}\right)}{\left(b_{3}-b_{1}\right) \eta-\left(a_{3}-a_{1}\right)}\right]+D_{3},
$$

where $D_{2}, D_{3} \in \mathbb{R}$.

c) If $\left(a_{3}, b_{3}\right)=(0,0)$, then $l_{3}=\lambda_{3}, l_{2}=\lambda_{3} \cosh \varphi(\eta), l_{1}=\lambda_{3} \sinh \varphi(\eta)$, with $\eta=\frac{a_{1} x_{1}+a_{2} x_{2}}{b_{1} x_{1}+b_{2} x_{2}}$ and $\varphi$ is given by

$$
\varphi(\eta)=\frac{E_{0}}{a_{2} b_{1}-a_{1} b_{2}} \arctan \left[\frac{b_{2}^{2}+b_{1}^{2}}{a_{2} b_{1}-a_{1} b_{2}}\left(\eta-\frac{a_{2} b_{2}+a_{1} b_{1}}{b_{2}^{2}+b_{1}^{2}}\right)\right]+E_{1},
$$

where $E_{0}, E_{1} \in \mathbb{R}$.

Proof. $a)$ If $\left(a_{1}, b_{1}\right)=(0,0)$ then Lemma 4 implies that $l_{1}=\lambda_{1}$ and Guichard condition implies that $l_{2}=\lambda_{1} \cosh \varphi(\eta)$ and $l_{3}=\lambda_{1} \sinh \varphi(\eta)$. In order to find $\varphi$, we use equation (3.10) with the following indices

$$
h_{32, x_{2}}+h_{23, x_{3}}+h_{31} h_{21}=0 .
$$


Since $h_{32}=\frac{\varphi_{, \eta} N_{2}}{\beta}, h_{23}=\frac{\varphi, \eta N_{3}}{\beta}$ and $h_{31}=h_{21}=0$, we rewrite the equation above as

$$
\left(\frac{\varphi, \eta N_{2}}{\beta}\right)_{, x_{2}}+\left(\frac{\varphi, \eta N_{3}}{\beta}\right)_{, x_{3}}=0
$$

By substituting the derivatives, we have the following ODE

$$
\varphi_{, \eta \eta}\left[\left(N_{2}\right)^{2}+\left(N_{3}\right)^{2}\right]-2 \varphi, \eta\left(b_{2} N_{2}+b_{3} N_{3}\right)=0,
$$

whose solution is exactly (3.39).

$b)$ If $\left(a_{2}, b_{2}\right)=(0,0)$, then $l_{2}=\lambda_{2}$ and Guichard condition implies that $l_{1}=\lambda_{2} \cos \varphi(\eta)$ and $l_{3}=\lambda_{2} \sin \varphi(\eta)$. In order to find $\varphi$, we use equation (3.10) with the following indices

$$
h_{13, x_{3}}+h_{31, x_{1}}+h_{12} h_{32}=0 .
$$

By using the same arguments as in $a$ ), we have the following ODE

$$
\varphi_{, \eta \eta}\left[\left(N_{1}\right)^{2}-\left(N_{3}\right)^{2}\right]-2 \varphi_{, \eta}\left(b_{1} N_{1}-b_{3} N_{3}\right)=0,
$$

whose solution will depend on $b_{1}$ and $b_{3}$. If $b_{1}=b_{3}$ we have $\varphi$ given by (3.40) and if $b_{1} \neq b_{3}$, the solution is given by (3.41).

$c$ ) The arguments when $\left(a_{3}, b_{3}\right)=(0,0)$ are the same as in $\left.a\right)$.

Remark 2. Although our calculation of the symmetry group for the Lamé's system has similar techniques to those used by Tenenblat and Winternitz for the intrinsic generalized wave and sine-Gordon equations in [24], we observe that the solutions invariant under the subgroups are quite different. In fact, when we consider the solutions invariant under the translation subgroup in Theorem 3, the solutions of (3.22) are given by Jacobi elliptic functions that cannot be reduced to elementary functions. Moreover, the only solutions of the Lamé's system, which are invariant under the action of the subgroup involving translation and dilations, that depend on all three variables are constant, in contrast to the solutions in [24]. The main reason is due to Guichard condition.

In the next two sections, we will deal with the geometric properties of the Guichard nets and of the conformally flat hypersurfaces associated to the solutions invariant under the 2dimensional translation subgroup. As we will see in Section 5, these are the solutions that will provide a new class of conformally flat hypersurfaces.

\section{Geometric properties of the Guichard nets}

In this section, we will study the geometric properties of the Guichard nets associated to locally conformally flat hypersurfaces corresponding to the solutions of the Lamé's system $l_{i}(\xi)$, which are invariant under the translation subgroup. Let $l_{1}(\xi), l_{2}(\xi), l_{3}(\xi)$, with $\xi=\sum_{s=1}^{3} \alpha_{s} x_{s}$ be a solution of Lamé's system. Theorem 1 implies that there is a Guichard net $x=\left(x_{1}, x_{2}, x_{3}\right)$ : $U \subset \mathbb{R}^{3} \rightarrow \mathbb{R}^{3}$, with a Riemannian metric

$$
g=l_{1}^{2} d x_{1}^{2}+l_{2}^{2} d x_{2}^{2}+l_{3}^{2} d x_{3}^{2},
$$

where $U$ is an open set, given by $U=\left\{\left(x_{1}, x_{2}, x_{3}\right) \in \mathbb{R}^{3} \mid \xi_{1}<\xi<\xi_{2}\right\}$, where $\xi_{1}$ and $\xi_{2}$ are real constants. 


\subsection{Level surfaces}

In this subsection, we will show that the Guichard nets are foliated by surfaces $\xi=\xi_{0}$ which are geodesically parallel. Moreover, we will prove that each such surface has flat Gaussian curvature and constant mean curvature that depends on $\xi_{0}$.

Definition 3. Let $M^{n}$ be a Riemannian manifold and let $f: M \rightarrow \mathbb{R}$ be a differentiable function. The level submanifolds of $f$ are said to be geodesically parallel if $|\operatorname{grad} f|$ is a non zero constant, along each level submanifold.

We have the following theorem

Theorem 6. Let $(U, g), U \subset \mathbb{R}^{3}$, be a Riemannian manifold with coordinates $\left(x_{1}, x_{2}, x_{3}\right)$ and metric $g=\sum_{s=1}^{3} l_{s}^{2}(\xi) d x_{i}^{2}$, where $\xi=\sum_{s=1}^{3} \alpha_{s} x_{s}$. Then the level surfaces

$$
P_{\xi_{0}}=\left\{\left(x_{1}, x_{2}, x_{3}\right) \in U ; \sum_{s=1}^{3} \alpha_{s} x_{s}=\xi_{0}\right\}, \quad \text { where } \xi_{1}<\xi_{0}<\xi_{2},
$$

endowed with the induced metric, are geodesically parallel. Moreover, each level surface has flat Gaussian curvature and constant mean curvature (depending on $\xi_{0}$ ).

Proof. Since at least one $\alpha_{i}$ is non zero, we can suppose that $\alpha_{3} \neq 0$ and we parametrize $P_{\xi_{0}}$ as

$$
X\left(x_{1}, x_{2}\right)=\left(x_{1}, x_{2}, \frac{\xi_{0}-\alpha_{1} x_{1}-\alpha_{2} x_{2}}{\alpha_{3}}\right) .
$$

Then $X_{x_{1}}=\left(1,0,-\alpha_{1} / \alpha_{3}\right)$ and $X_{x_{2}}=\left(0,1,-\alpha_{2} / \alpha_{3}\right)$. Consequently, the coefficients of the induced metric are constant, since $\xi=\xi_{0}$ in this surface. Therefore the Gaussian curvature is equal to zero.

Consider now the function $h(x)=\sum_{i=1}^{3} \alpha_{i} x_{i}$. Then $P_{\xi_{0}}=h^{-1}\left(\xi_{0}\right)$. Since $h$ is constant along $P_{\xi_{0}}$, it follows that $\operatorname{grad} h$ is normal to $P_{\xi_{0}}$. Moreover,

$$
g(\operatorname{grad} h, \operatorname{grad} h)=\sum_{j=1}^{3} \frac{\alpha_{j}^{2}}{l_{j}^{2}}
$$

which implies that $|\operatorname{grad} h|$ is constant along $P_{\xi_{0}}$. It follows from Definition 3 that the level surfaces $h^{-1}\left(\xi_{0}\right)$ are geodesically parallel.

Now we compute the mean curvatures of $P_{\xi_{0}}$. Given $p \in P_{\xi_{0}}$, let $A: T_{p} P_{\xi_{0}} \rightarrow T_{p} P_{\xi_{0}}$ be the Weingarten operator, i.e., $A \mathbf{v}=-\nabla_{\mathbf{v}}\left(\frac{\operatorname{grad} h}{|\operatorname{grad} h|}\right)(p)$, where $\nabla$ is the Riemannian connection on $(U, g)$. Since $|\operatorname{grad} h|$ is constant along $P_{\xi_{0}}$, it follows that

$$
A \mathbf{v}=-\frac{1}{|\operatorname{grad} h|} \nabla_{\mathbf{v}} \operatorname{grad} h(p) .
$$

Then the mean curvature of $P_{\xi_{0}}$ is given by

$$
H=-\frac{\Delta h(p)}{|\operatorname{grad} h|}=\frac{1}{|\operatorname{grad} h|} \sum_{i, k} \frac{\Gamma_{i i}^{k}\left(\xi_{0}\right) \alpha_{k}}{l_{i}^{2}\left(\xi_{0}\right)}
$$

where $\Gamma_{i j}^{k}$ are the Christoffel for the connection $\nabla$. Therefore, the mean curvature of $P_{\xi_{0}}$ is a constant depending on $\xi_{0}$. 


\subsection{Coordinate surfaces}

In this subsection, we will use the solutions invariant by the group of translations to show that the coordinate surfaces of the corresponding Guichard net $(U, g)$ have constant Gaussian curvature. Moreover, the values of these curvatures satisfy an algebraic relation.

Theorem 7. Let $(U, g), U \subset \mathbb{R}^{3}$, be a Riemannian manifold, with coordinates $\left(x_{1}, x_{2}, x_{3}\right)$ and metric $g=\sum_{s=1}^{3} l_{s}^{2}(\xi) d x_{i}^{2}$, with $\xi=\sum_{s=1}^{3} \alpha_{s} x_{s}$. Then each coordinate surface of $U \subset \mathbb{R}^{3}, x_{i}=$ const, endowed with the induced metric, has constant Gaussian curvature $K_{i}$. Moreover,

$$
K_{1}+K_{2}+K_{3}=0
$$

Proof. Since $g$ is given by (4.1), it follows that the metric induced on each coordinate surface, $x_{i}=$ const, is

$$
g_{i}=l_{j}^{2}\left(d x_{j}\right)^{2}+l_{k}^{2}\left(d x_{k}\right)^{2}, \quad i, j, k \text { distinct },
$$

and its Gaussian curvature, $K_{i}$, is given by

$$
K_{i}=\frac{1}{l_{j} l_{k}}\left(\frac{l_{k, x_{i}} l_{j, x_{i}}}{l_{i}^{2}}\right) .
$$

Assume that none of the functions $l_{i}$ is constant and $\xi=\alpha_{1} x_{1}+\alpha_{2} x_{2}+\alpha_{3} x_{3}$, with $\alpha_{i} \neq 0$, for all $i$. In this case, we have $l_{i, \xi}=c_{i} l_{j} l_{k}$, where $i, j$ and $k$ are distinct indices in $\{1,2,3\}$. Therefore, it follows from (4.2) that the Gaussian curvature of each coordinate surface is given by $K_{i}=c_{j} c_{k} \alpha_{i}^{2}$. Moreover, it follows from (3.21) that

$$
K_{1}+K_{2}+K_{3}=\alpha_{1}^{2} c_{2} c_{3}+\alpha_{2}^{2} c_{3} c_{1}+\alpha_{1}^{3} c_{1} c_{2}=0 .
$$

If only one of the functions $l_{i}$ is constant, it follows from Lemma 2, that, if $l_{i}$ is constant, then $\alpha_{i}=0$. Then it follows from (4.2) that all the curvatures are equal to zero. In fact, $K_{i}=0$, since the functions $l_{s}$, for all $s$, do not depend on $x_{i}$. Moreover, for $j \neq i, K_{j}=0$, since $l_{i}$ is constant. Hence, the sum $\sum_{j=1}^{3} K_{i}=0$ trivially.

\section{Conformally flat hypersurfaces}

In this section, we describe the generic conformally flat hypersurfaces associated to the solutions of the Lamé's system invariant under the translation group.

It is known that, any locally generic conformally flat hypersurface, in a 4-dimensional space form, has a metric induced by the Guichard net of the form (see [10, 21, 22])

$$
g=e^{2 P(x)}\left\{\sin ^{2} \varphi(x) d x_{1}^{2}+d x_{2}^{2}+\cos ^{2} \varphi(x) d x_{3}^{2}\right\},
$$

where $x=\left(x_{1}, x_{2}, x_{3}\right)$, or

$$
g=e^{2 \tilde{P}(x)}\left\{\sinh ^{2} \tilde{\varphi}(x) d x_{1}^{2}+\cosh ^{2} \tilde{\varphi}(x) d x_{2}^{2}+d x_{3}^{2}\right\} .
$$

Suyama classified in [22] the hypersurfaces conformal to the products $M^{2} \times I \subset \mathbb{R}^{4}$ given by Lafontaine in [13], as the hypersurfaces where $\varphi$ depends only on two variables. Hertrich-Jeromin and Suyama classified in [10] the hypersurfaces where $\varphi$ has two vanishing mixed derivatives. These conformally flat hypersurfaces are associated to the so called cyclic Guichard nets, which are characterized by $\varphi_{, x_{1} x_{2}}=\varphi_{, x_{2} x_{3}}=0$, when $g$ is of the form (5.1) and by $\varphi_{, x_{1} x_{3}}=\varphi_{, x_{2} x_{3}}=0$, 
when $g$ is given by (5.2). Moreover, the authors showed that all the known cases of conformally flat hypersurfaces, up to now, are associated to cyclic Guichard nets.

We observe that Theorem 5 shows that each solution of the Lamé's system, which is invariant under the action of the 2-dimensional subgroup involving translations and dilations, depends only on two variables. Therefore, the conformally flat hypersurfaces associated to these solutions are conformal to the products $M^{2} \times I \subset \mathbb{R}^{4}$.

We now consider the conformally flat hypersurfaces associated to the solutions invariant under the translation subgroup. We analyse each case separately:

i) $\xi=\alpha_{1} x_{1}+\alpha_{2} x_{2}$. In this case, we have the solutions $l_{1}=\lambda_{3} \sinh \left(\xi+\xi_{0}\right), l_{2}=\lambda_{3} \cosh \left(\xi+\xi_{0}\right)$ and $l_{3}=\lambda_{3} \neq 0$ (see Theorem 4 ). Then the associated conformally flat hypersurface has a Guichard net, where the induced metric is given by

$$
g=e^{2 P\left(x_{1}, x_{2}, x_{3}\right)}\left\{\sinh ^{2}\left(\xi+\xi_{0}\right) d x_{1}^{2}+\cosh ^{2}\left(\xi+\xi_{0}\right) d x_{2}^{2}+d x_{3}^{2}\right\} .
$$

The hypersurface is conformal to one of the products considered by Lafontaine in [13] that we describe as follows (see $[21,22]$ for details): Let $\mathbb{H}^{3}$ be the hyperbolic 3 -space, considered as the half space model and as a subset of $\mathbb{R}^{4}$, i.e.,

$$
\mathbb{H}^{3}=\left\{\left(y^{1}, y^{2}, y^{3}, 0\right): y^{3}>0\right\} \subset \mathbb{R}^{4}=\left\{\left(y^{1}, y^{2}, y^{3}, y^{4}\right): y^{i} \in \mathbb{R}\right\},
$$

with the metric $g_{i j}=\frac{\delta_{i j}}{y_{3}^{2}}$. Consider the rotations of the $y^{3}$-axis given by

$$
\left(y^{1}, y^{2}, y^{3}, 0\right) \rightarrow\left(y^{1}, y^{2}, y^{3} \cos t, y^{3} \sin t\right)
$$

then the hypersurface $M^{3}=M^{2} \times I$, obtained by the above rotation of a surface of constant curvature $M^{2} \subset \mathbb{H}^{3}$ is a conformally flat hypersurface. One can show that for $g$ given by (5.3), the surface $M^{2} \subset \mathbb{H}^{3}$ is a flat surface, parametrized by lines of curvature whose first and second fundamental forms are given by

$$
\begin{aligned}
& I=\sinh ^{2}\left(\xi+\xi_{0}\right) d x_{1}^{2}+\cosh ^{2}\left(\xi+\xi_{0}\right) d x_{2}^{2}, \\
& I I=\sinh \left(\xi+\xi_{0}\right) \cosh \left(\xi+\xi_{0}\right)\left(d x_{1}^{2}+d x_{2}^{2}\right) .
\end{aligned}
$$

In order to describe the flat surfaces $M^{2} \subset \mathbb{H}^{3}$, we mention a classification result obtained by the authors in collaboration with Martínez [16]. It is well known that, on a neighbourhood of a non-umbilical point, a flat surface in $\mathbb{H}^{3}$ can be parametrized by lines of curvature, so that the first and second fundamental forms are given by (for details, see [23, Theorem 2.4, Corollary 2.7])

$$
\begin{aligned}
& I=\sinh ^{2} \phi(u, v)(d u)^{2}+\cosh ^{2} \phi(u, v)(d v)^{2}, \\
& I I=\sinh \phi(u, v) \cosh \phi(u, v)\left((d u)^{2}+(d v)^{2}\right),
\end{aligned}
$$

where $\phi$ is a harmonic function, i.e. $\phi_{u u}+\phi_{v v}=0$. The classification result is given as follows:

Theorem 8 ([16]). Let $\Sigma$ be a flat surface in $\mathbb{H}^{3}$ with a local parametrization, in a neighborhood of a nonsingular and nonumbilic point, such that the first and second fundamental forms are diagonal and given by (5.5) and (5.6), where $\phi$ is a (Euclidean) harmonic function. Then $\phi$ is linear, i.e., $\phi=a u+b v+c$ if, and only if, $\Sigma$ is locally congruent to either a helicoidal flat surface (when $(a, b, c) \neq(0, \pm 1,0))$ or to a "peach front" (when $(a, b, c)=(0, \pm 1,0))$.

Helicoidal surfaces arise as a natural generalization of rotational surfaces. They are invariant by a helicoidal group of isometries, i.e., given an axis, we consider a translation along this axis composed with a rotation around it. In the half space model of $\mathbb{H}^{3}$, up to isometries, we can 
consider the $y_{3}$-axis, which enables us to write the helicoidal group, relative to this axis, as the composition

$$
h_{t}=\left(\begin{array}{ccc}
e^{\beta t} & 0 & 0 \\
0 & e^{\beta t} & 0 \\
0 & 0 & e^{\beta t}
\end{array}\right)\left(\begin{array}{ccc}
\cos \alpha t & -\sin \alpha t & 0 \\
\sin \alpha t & \cos \alpha t & 0 \\
0 & 0 & 1
\end{array}\right),
$$

of a rotation around the $y_{3}$-axis with angular pitch $\alpha$ with a hyperbolic translation of ratio $\beta$. The "peach front" is a special case of flat surfaces that is not helicoidal. Details about this surface can be found in [11].

The study of flat surfaces in hyperbolic 3-space has received a lot of attention in the last few years, mainly because Galvéz, Martínez and Milán have shown in [5] that flat surfaces in the hyperbolic 3-space admit a Weierstrass representation formula in terms of meromorphic data as in the theory of minimal surfaces in $\mathbb{R}^{3}$. Namely, if $\psi: M^{2} \rightarrow \mathbb{H}^{3}$ is a surface in $\mathbb{H}^{3}$, for any $p \in M^{2}$, there exist $G(p), G^{*}(p) \in \mathbb{C}_{\infty}$ distinct points in the ideal boundary of $\mathbb{H}^{3}$ such that the oriented normal geodesic at $\psi(p)$ is the geodesic in $\mathbb{H}^{3}$ starting from $G^{*}(p)$ towards $G(p)$. The maps $G, G^{*}: \Sigma \rightarrow \mathbb{C}_{\infty}$ are called the hyperbolic Gauss maps of $\psi$. It is proved in [5] that, for flat surfaces, they are holomorphic when one considers $\mathbb{C}_{\infty}$ as the Riemann sphere and $M^{2}$ has a complex structure induced by the second fundamental form. Conversely, given two holomorphic functions $G$ and $G^{*}$, with $G \neq G^{*}$, one can recover a flat immersion of a surface in $\mathbb{H}^{3}$ (for more details see also $[3,11,12]$ ). This representation formula was the main tool to obtain Theorem 8 .

With the previous results we conclude that

Theorem 9. Let $l_{i}(\xi)$ be solutions of the Lamés system, where $\xi=\alpha_{1} x_{1}+\alpha_{2} x_{2}$. Then the associated conformally flat hypersurfaces are conformal to the product, $M^{2} \times I$, where $M^{2}$ is locally congruent to either a helicoidal flat surface in $\mathbb{H}^{3}$ or the "peach front".

Proof. When $\xi=\alpha_{1} x_{1}+\alpha_{2} x_{2}$, it follows from Theorem 4 that the solution of Lamé's system is $l_{1}=\lambda_{3} \sinh \left(\xi+\xi_{0}\right), l_{2}=\lambda_{3} \cosh \left(\xi+\xi_{0}\right)$ and $l_{3}=\lambda_{3} \neq 0$ and the corresponding conformally flat hypersurface $M^{3}$ has a metric $g$ given by (5.3). Then $M^{3}$ is conformal to the product $M^{2} \times I$, where $M^{2}$ is a flat surface in $\mathbb{H}^{3}$ with fundamental forms given by (5.4). It follows from Theorem 8 that $M^{2}$ is locally congruent to either a helicoidal flat surface in $\mathbb{H}^{3}$ or to the "peach front".

ii) $\xi=\alpha_{1} x_{1}+\alpha_{3} x_{3}$. In this case, we have the solution of Lamé's system, $l_{1}=\lambda_{2} \sin \left(\xi+\xi_{0}\right)$, $l_{2}=\lambda_{2} \neq 0$ and $l_{3}=\lambda_{2} \cos \left(\xi+\xi_{0}\right)$ (see Theorem $\left.4 \mathrm{~b}\right)$ ). Then the associated conformally flat hypersurface $M^{3}$ has a Guichard net, whose induced metric is given by

$$
g=e^{2 P\left(x_{1}, x_{2}, x_{3}\right)}\left\{\sin ^{2}\left(\xi+\xi_{0}\right) d x_{1}^{2}+d x_{2}^{2}+\cos ^{2}\left(\xi+\xi_{0}\right) d x_{3}^{2}\right\} .
$$

The hypersurface $M^{3}$ is conformal to another class of products $M^{2} \times I$ (see [21, 22]). Namely, let $\mathbb{S}^{3} \subset \mathbb{R}^{4}$ be the canonical 3-sphere, then $M^{2} \times I=\left\{t p: 0<t<\infty, p \in M^{2} \subset \mathbb{S}^{3}\right\}$, is a conformally flat hypersurface, where $M^{2}$ is a surface with constant curvature in $S^{3}$. In our case, $M^{3}$ is conformal to the product $M^{2} \times I$, where the surface $M^{2} \subset \mathbb{S}^{3}$ is a flat surface, parametrized by lines of curvature, whose first and second fundamental forms are given by

$$
\begin{aligned}
& I=\sin ^{2}\left(\xi+\xi_{0}\right) d x_{1}^{2}+\cos ^{2}\left(\xi+\xi_{0}\right) d x_{3}^{2}, \\
& I I=\sin \left(\xi+\xi_{0}\right) \cos \left(\xi+\xi_{0}\right)\left(d x_{1}^{2}-d x_{3}^{2}\right) .
\end{aligned}
$$

The geometry of these surfaces in $\mathbb{S}^{3}$ is being studied and it will appear in another paper.

iii) $\xi=\alpha_{1} x_{1}+\alpha_{2} x_{2}+\alpha_{3} x_{3}, \alpha_{i} \neq 0$, for all $i$. In this case, we will show that the solutions $l_{i}(\xi)$ of the Lamé's system give rise to a new class of conformally flat hypersurfaces, according to the following theorem: 
Theorem 10. Let $M^{3}$ be a conformally flat hypersurface in a space form $M_{K}^{4}$, associated to a solution of Lamé's system $l_{i}\left(x_{1}, x_{2}, x_{3}\right)=l_{i}(\xi)$, with $\xi=\sum_{s=1}^{3} \alpha_{s} x_{s}$ and $\alpha_{s} \neq 0$, for all s, given in terms of elliptic functions by (3.22)-(3.24). Then its first fundamental form $g$ is given by

$$
g=e^{2 P(x)}\left\{\cos ^{2} \varphi(\xi)\left(d x_{1}\right)^{2}+\left(d x_{2}\right)^{2}+\sin ^{2} \varphi(\xi)\left(d x_{3}\right)^{2}\right\}
$$

where $\varphi$ satisfies,

$$
\varphi_{, \xi}^{2}=c\left(a \cos ^{2} \varphi-b\right),
$$

or $g$ is given by

$$
g=e^{2 \tilde{P}(x)}\left\{\sinh ^{2} \tilde{\varphi}(\xi)\left(d x_{1}\right)^{2}+\cosh ^{2} \tilde{\varphi}(\xi)\left(d x_{2}\right)^{2}+\left(d x_{3}\right)^{2}\right\}
$$

where $\tilde{\varphi}$ satisfies

$$
\tilde{\varphi}_{, \xi}^{2}=c\left(b \cosh ^{2} \tilde{\varphi}-b\right) .
$$

where $a, b, c \in \mathbb{R} \backslash\{0\}, P(x)$ and $\tilde{P}(x)$ are differentiable functions that depend on $l_{s}$ and $M_{K}^{4}$. In both cases, $\xi \in I \subset \mathbb{R}$, where $I$ is an open interval such that $g$ is positive definite.

Proof. Guichard condition (2.1) implies that we may consider

$$
\begin{aligned}
& l_{1}=l_{2} \cos \varphi, \\
& l_{3}=l_{2} \sin \varphi .
\end{aligned}
$$

It follows from Theorem 1 that the metric is given by (5.8). In order to obtain the expression for the derivative of $\varphi$ with respect to $\xi$, we consider

$$
l_{1, \xi}=l_{2, \xi} \cos \varphi-l_{2} \varphi_{, \xi} \sin \varphi .
$$

Since $\alpha_{s} \neq 0$ for all $s$, the functions $l_{i}$ are given as in Theorem 3, by (3.22)-(3.24). Hence, using (3.19), we have that

$$
c_{1} l_{2} l_{3}=c_{2} l_{1} l_{3} \cos \varphi-\varphi_{, \xi} l_{3},
$$

for $c_{1}, c_{2} \in \mathbb{R} \backslash\{0\}$, which implies

$$
\varphi_{, \xi}=l_{2}\left(c_{2} \cos ^{2} \varphi-c_{1}\right) .
$$

By taking the derivative again, it follows from (5.12)-(5.14) and (3.19) that

$$
\begin{aligned}
\varphi_{, \xi \xi} & =l_{2, \xi}\left(c_{2} \cos ^{2} \varphi-1\right)-2 c_{2} l_{2} \cos \varphi \sin \varphi \varphi_{, \xi}=\frac{1}{l_{2}}\left[l_{2, \xi} \varphi_{, \xi}-2 c_{2}\left(l_{2} \cos \varphi\right)\left(l_{2} \sin \varphi\right) \varphi_{, \xi}\right] \\
& =\frac{1}{l_{2}}\left[l_{2, \xi} \varphi_{, \xi}-2 c_{2} l_{1} l_{3} \varphi_{, \xi}\right]=\frac{1}{l_{2}}\left[l_{2, \xi} \varphi_{, \xi}-2 l_{2, \xi} \varphi_{, \xi}\right]=-\frac{l_{2, \xi} \varphi_{, \xi}}{l_{2}} .
\end{aligned}
$$

Therefore,

$$
\varphi, \xi l_{2}=c,
$$

where $c \in \mathbb{R} \backslash\{0\}$, since $\varphi_{, \xi} \neq 0$. Then, multiplying (5.14) by $\varphi_{, \xi}$ and using (5.15) we have that $\varphi_{, \xi}^{2}=c\left(c_{2} \cos ^{2} \varphi-c_{1}\right)$, i.e., (5.9) holds. The proof of the second part of the theorem is analogous, when we consider $l_{2}=l_{3} \cosh \varphi$ and $l_{1}=l_{3} \sinh \varphi$. 
Corollary 2. Let $M^{3} \subset M^{4}$ be a conformally flat hypersurface associated to the solutions of Lamé's system $l_{i}(\xi)$ with $\xi=\sum_{s=1}^{3} \alpha_{s} x_{s}$ and $\alpha_{s} \neq 0$ for all $s$, given in terms of elliptic functions by (3.22)-(3.24). Then the Guichard net of $M^{3}$ is not cyclic.

Proof. It follows from Theorem 10 that the first fundamental form of $M^{3}$ is given by (5.8), where $\varphi(\xi)$ satisfies $(5.9)$ or by (5.10) where $\tilde{\varphi}(\xi)$ satisfies $(5.11), \xi \in I \subset \mathbb{R}$. In the first case, (5.9) implies that $\varphi_{, \xi \xi}=\lambda \sin 2 \varphi, \lambda \neq 0$ and in the second case, (5.11) implies that $\tilde{\varphi}_{, \xi \xi}=\lambda \sin 2 \tilde{\varphi}, \lambda \neq 0$. In either case, $\varphi_{, x_{i} x_{j}}=\alpha_{i} \alpha_{j} \varphi_{, \xi \xi} \neq 0, i \neq j$ and $\xi \in I$. Therefore, the Guichard net of $M^{3}$ is not cyclic.

We observe that, as a consequence of the results of Hertrich-Jeromin and Suyama, the surfaces $M^{3}$ of Corollary 2 provide a new class of conformally flat hypersurfaces.

It is important to observe that Hertrich-Jeromin and Suyama in [9] have independently considered Guichard nets with the ansatz on the function $\varphi$ such that $\varphi\left(x_{1}, x_{2}, x_{3}\right)=\varphi\left(a x_{1}+\right.$ $\left.b x_{2}+c x_{3}\right)$. They investigated the geometric properties of these Guichard nets, that they called Bianchi-type Guichard nets, as well as the new class of associated conformally flat hypersurfaces.

\section{A Appendix}

Proof of Theorem 2. The infinitesimal generator associated to the symmetry group is written as in (3.12). The functions $\xi^{i}, \eta^{i}, \phi^{i j}$ will be obtained by solving the determining equations that arise when we apply the first prolongation formula

$$
\begin{aligned}
\operatorname{pr}^{(1)} V= & V+\sum_{i, k} D_{k}\left(\eta^{i}\right) \frac{\partial}{\partial l_{i, x_{k}}}+\sum_{i, j, k i \neq j} D_{k}\left(\phi^{i j}\right) \frac{\partial}{\partial h_{i j, x_{k}}} \\
& -\sum_{i, k, r} D_{k}\left(\xi^{r}\right) l_{i, x_{r}} \frac{\partial}{\partial l_{i, x_{k}}}-\sum_{i, j, k, r} D_{k}\left(\xi^{r}\right) h_{i j, x_{r}} \frac{\partial}{\partial h_{i j, x_{k}}},
\end{aligned}
$$

with

$$
D_{i}=\frac{\partial}{\partial x_{i}}+\sum_{j} l_{j, x_{i}} \frac{\partial}{\partial l_{i}}+\sum_{j, l} h_{j l, x_{i}} \frac{\partial}{\partial h_{j l}},
$$

on each equation of the system, i.e., when we consider the infinitesimal criterion (3.2). In order to avoid any functional dependence, the following substitutions will be considered

$$
\begin{aligned}
& l_{i, x_{j}}=h_{i j} l_{j}, \quad i \neq j, \\
& l_{i, x_{i}}=-\varepsilon_{i} \varepsilon_{j} h_{j i} l_{j}-\varepsilon_{i} \varepsilon_{k} h_{k i} l_{k} \\
& h_{i j, x_{k}}=h_{i k} h_{k j}, \\
& h_{i j, x_{j}}=-h_{j i, x_{i}}-h_{i k} h_{j k}, \quad i<j, \quad \\
& h_{i j, x_{i}}=-\varepsilon_{i} \varepsilon_{j} h_{j i, x_{j}}-\varepsilon_{i} \varepsilon_{k} h_{k i} h_{k j}, \quad i<j .
\end{aligned}
$$

Fixing $i, j$ and $k$, distinct indices, we start applying $\operatorname{pr}^{(1)} V$ to equation (3.9). Then the infinitesimal criterion (3.2), gives $\phi_{(k)}^{i j}-\phi^{i k} h_{k j}-h_{i k} \phi^{k j}=0$, using the prolongation formula, we get

$$
\begin{aligned}
\phi_{, x_{k}}^{i j} & +\sum_{r} \phi_{, l_{r}}^{i j} l_{r, x_{k}}+\sum_{r, s} \phi_{, h_{r s}}^{i j} h_{r s, x_{k}}-\sum_{t}\left(\xi_{, x_{k}}^{t}+\sum_{r} \xi_{, l_{r}}^{t} l_{r, x_{k}}+\sum_{r, s} \xi_{, h_{r s}}^{t} h_{r s, x_{k}}\right) h_{i j, x_{t}} \\
& -\phi^{i k} h_{k j}-h_{i k} \phi^{k j}=0 .
\end{aligned}
$$


For $i<j$, we apply the substitutions (A.1)-(A.5) and we analyse each term of (A.6) as follows

$$
\begin{aligned}
& \sum_{r} \phi_{, l_{r}}^{i j} l_{r, x_{k}}=\sum_{r \neq k} \phi_{, l_{r}}^{i j} h_{r k} l_{k}-\phi_{, l_{k}}^{i j}\left(\varepsilon_{k} \varepsilon_{j} h_{j k} l_{j}+\varepsilon_{k} \varepsilon_{i} h_{i k} l_{i}\right), \\
& \begin{aligned}
& \sum_{r, s} \phi_{, h_{r s}}^{i j} h_{r s, x_{k}}= \sum_{r \neq k, s \neq k} \phi_{, h_{r s}}^{i j} h_{r k} h_{k s}+\sum_{s<k} \phi_{, h_{k s}}^{i j} h_{k s, x_{k}}-\sum_{s>k} \phi_{, h_{k s}}^{i j}\left(\varepsilon_{k} \varepsilon_{s} h_{s k, x_{s}}+\varepsilon_{k} \varepsilon_{m} h_{m k} h_{m s}\right) \\
&-\sum_{r<k} \phi_{, h_{r k}}^{i j}\left(h_{k r, x_{r}}+h_{r n} h_{k n}\right)+\sum_{r>k} \phi_{, h_{r k}}^{i j} h_{r k, x_{k}}, \\
& \sum_{t}\left(\xi_{, x_{k}}^{t}+\sum_{r} \xi_{, l_{r}}^{t} l_{r, x_{k}}+\sum_{r, s} \xi_{, h_{r s}}^{t} h_{r s, x_{k}}\right) h_{i j, x_{t}} \\
&=C_{k}^{k} h_{i k} h_{k j}-C_{k}^{j}\left(h_{j i, x_{i}}+h_{i k} h_{j k}\right)-C_{k}^{i}\left(\varepsilon_{i} \varepsilon_{j} h_{j i, x_{j}}+\varepsilon_{i} \varepsilon_{k} h_{k i} h_{k j}\right),
\end{aligned}
\end{aligned}
$$

where the coefficients $C_{k}^{t}$ are given by

$$
\begin{aligned}
C_{k}^{t}= & \xi_{, x_{k}}^{t}+\sum_{r \neq k} \xi_{, l_{r}}^{t} h_{r k} l_{k}-\xi_{, l_{k}}^{t}\left(\varepsilon_{k} \varepsilon_{j} h_{j k} l_{j}+\varepsilon_{k} \varepsilon_{i} h_{i k} l_{i}\right)+\sum_{r \neq k, s \neq k} \xi_{, h_{r s}}^{t} h_{r k} h_{k s} \\
& +\sum_{s<k} \xi_{, h_{k s}}^{t} h_{k s, x_{k}}-\sum_{s>k} \xi_{, h_{k s}}^{t}\left(\varepsilon_{k} \varepsilon_{s} h_{s k, x_{s}}+\varepsilon_{k} \varepsilon_{m} h_{m k} h_{m s}\right) \\
& -\sum_{r<k} \xi_{, h_{r k}}^{t}\left(h_{k r, x_{r}}+h_{r n} h_{k n}\right)+\sum_{r>k} \xi_{, h_{r k}}^{t} h_{r k, x_{k}},
\end{aligned}
$$

and the indices $m$ and $n$ are such that $\{k, s, m\}, s>k$ and $\{k, r, n\}, r<k$ are two sets of three distinct numbers.

Now we analyse the coefficients of equation (A.6), considering (A.7)-(A.9). By equating to zero the coefficients of the products $h_{j i, x_{j}} h_{k s, x_{k}}$, with $k>s$, we obtain $\xi_{, h_{k s}}^{i}=0$. Analogously, for the coefficients of $h_{j i, x_{j}} h_{s k, x_{s}}$, with $k<s$, we obtain $\xi_{, h_{k s}}^{i}=0$. This implies that

$$
\xi_{, h_{k s}}^{i}=0, \quad \forall s, \quad s \neq k, \quad \text { i.e. } \quad \xi_{, h_{k j}}^{i}=\xi_{, h_{k i}}^{i}=0 .
$$

Similarly, from the coefficients of $h_{j i, x_{j}} h_{k r, x_{k}}, r<k$ and $h_{j i, x_{j}} h_{r k, x_{k}}$, with $r>k$, we obtain

$$
\xi_{, h_{r k}}^{i}=0, \quad \forall r, \quad r \neq k, \quad \text { i.e. } \quad \xi_{, h_{j k}}^{i}=\xi_{, h_{i k}}^{i}=0,
$$

where $i, j, k \in\{1,2,3\}$ are distinct and $i<j$. By analysing the coefficients of $h_{j i, x_{i}} h_{k s, x_{k}}$ with $k>s$ and $h_{j i, x_{i}} h_{s k, x_{s}}$ with $k<s$, we obtain

$$
\xi_{, h_{k s}}^{j}=0, \quad \forall s, \quad s \neq k, \quad \text { i.e. } \quad \xi_{, h_{k i}}^{j}=\xi_{, h_{k j}}^{j}=0 .
$$

Similarly, the coefficients of $h_{j i, x_{i}} h_{k r, x_{r}}$, with $k>r$, and $h_{j i, x_{i}} h_{r k, x_{k}}$, with $k<r$, lead to

$$
\xi_{, h_{r k}}^{j}=0, \quad \forall r, \quad r \neq k, \quad \text { i.e. } \quad \xi_{, h_{i k}}^{j}=\xi_{, h_{j k}}^{j}=0 .
$$

Since $i, j, k \in\{1,2,3\}$ are distinct and arbitrary indices, with $i<j$, we conclude that $\xi_{, h_{s t}}^{m}=0$ for any indices $m, s$ and $t, s \neq t$, i.e., $\xi^{m}$ depends only on $x$ and $l$. Therefore, the expression of $C_{k}^{t}$ given in (A.10) reduces to

$$
C_{k}^{t}=\xi_{, x_{k}}^{t}+\sum_{r \neq k} \xi_{, l_{r}}^{t} h_{r k} l_{k}-\xi_{, l_{k}}^{t}\left(\varepsilon_{k} \varepsilon_{j} h_{j k} l_{j}+\varepsilon_{k} \varepsilon_{i} h_{i k} l_{i}\right)
$$

that can be rewritten as

$$
C_{k}^{t}=\xi_{, x_{k}}^{t}+\sum_{r \neq k}\left(\xi_{, l_{r}}^{t} l_{k}-\xi_{, l_{k}}^{t} \varepsilon_{r} \varepsilon_{k} l_{r}\right) h_{r k} .
$$


From (A.8), we have that the coefficients of $h_{k s, x_{k}}$, with $s<k$, and the coefficients of $h_{s k, x_{s}}$, with $s>k$, lead to $\phi_{, h_{k s}}^{i j}=0, \forall s \neq k$, i.e. $\phi_{, h_{k i}}^{i j}=\phi_{, h_{k j}}^{i j}=0$.

Considering (A.9), the coefficients of $h_{j i, x_{i}}$ and $h_{j i, x_{j}}$ imply that $C_{k}^{j}=0$ and $C_{k}^{i}=0$, respectively. Since $i<j$ and $i, j, k \in\{1,2,3\}$ are arbitrary and distinct, we conclude that $C_{k}^{i}=C_{k}^{j}=0$, for all $i, j, k$ distinct indices.

Since $\xi^{m}$ does not depend on $h_{s t}$, the analysis of (A.11) gives us the following system

$$
\begin{aligned}
& \xi_{, x_{k}}^{m}=0, \quad \forall m \neq k, \\
& \xi_{l_{r}}^{m} l_{k}-\varepsilon_{r} \varepsilon_{k} \xi_{, l_{k}}^{m} l_{r}=0, \quad \forall r \neq k .
\end{aligned}
$$

The first equation of this system says that $\xi^{m}$ depends only on $x_{m}$ and $l$. By solving the characteristic system for the second equation, we have that $\xi^{m}$ depends on $x_{m}$ and a variable $\zeta=\varepsilon_{i} l_{i}^{2}+\varepsilon_{j} l_{j}^{2}+\varepsilon_{k} l_{k}^{2}$. However, Guichard condition implies that $\zeta \equiv 0$, hence $\xi^{m}$ does not depend on $l_{s}$, for all $s$.

Summarising the conclusions of this first part of the proof, we have that

$$
\phi^{s t}=\phi^{s t}\left(h_{s t}, h_{t s}, x, l\right), \quad \text { and } \quad \xi^{m}=\xi^{m}\left(x_{m}\right) .
$$

We now consider equation (3.7). By applying the prolongation $\operatorname{pr}^{(1)} V$ to (3.7), we have that $\eta_{(j)}^{i}-\phi^{i j} l_{j}-h_{i j} \eta^{j}=0$, which implies,

$$
\eta_{, x_{j}}^{i}+\sum_{r} \eta_{, l_{r}}^{i} l_{r, x_{j}}+\sum_{r, s} \eta_{, h_{r s}}^{i} h_{r s, x_{j}}-\xi_{, x_{j}}^{j} l_{i, x_{j}}-\phi^{i j} l_{j}-h_{i j} \eta^{j}=0 .
$$

Observe that by applying the substitution (A.1), we have

$$
\sum_{r} \eta_{, l_{r}}^{i} l_{r, x_{j}}=\sum_{r \neq j} \eta_{, l_{r}}^{i} h_{r j} l_{j}-\eta_{, l_{j}}^{i}\left(\varepsilon_{j} \varepsilon_{i} h_{i j} l_{i}+\varepsilon_{j} \varepsilon_{k} h_{k j} l_{k}\right)
$$

Moreover, by applying the substitutions (A.3), (A.4) and (A.5) we have

$$
\begin{aligned}
\sum_{r, s} \eta_{, h_{r s}}^{i} h_{r s, x_{j}}= & \sum_{r \neq j, s \neq j} \eta_{, h_{r s}}^{i} h_{r j} h_{j s}+\sum_{s<j} \eta_{, h_{j s}}^{i} h_{j s, x_{j}}-\sum_{s>j}\left(\varepsilon_{j} \varepsilon_{s} h_{s j, x_{s}}+\varepsilon_{j} \varepsilon_{m} h_{m j} h_{m s}\right) \\
& -\sum_{r<j} \eta_{,_{r j}}^{i}\left(h_{j r, x_{r}}+h_{r n} h_{j n}\right)+\sum_{r>j} \eta_{,_{r j}}^{i} h_{r j, x_{j}} .
\end{aligned}
$$

Therefore, by considering in (A.12), the coefficients of $h_{j s, x_{j}}$, with $s<j$, and $h_{s j, x_{s}}$, with $s>j$, we conclude that $\eta_{, h_{j s}}^{i}=0$. Similarly, the analysis of the coefficients of $h_{j r, x_{r}}$, with $r<j$, and $h_{r j, x_{j}}$, with $r>j$, imply that $\eta_{, h_{r j}}^{i}=0$. Hence, we conclude that

$$
\eta_{, h_{j t}}^{i}=\eta_{, h_{t j}}^{i}=0, \quad \forall t \neq j .
$$

Since $i$ and $t \neq j$ are arbitrary, we conclude that $\eta^{m}$ does not depend on $h_{s t}$, for any indices, $m, s$ and $t$ with $s \neq t$. Consequently, (A.12) reduces to

$$
\eta_{, x_{j}}^{i}+\left(\eta_{, l_{i}}^{i} l_{j}-\varepsilon_{i} \varepsilon_{j} \eta_{, l_{j}}^{i} l_{i}-\xi_{, x_{j}}^{j} l_{j}-\eta^{j}\right) h_{i j}+\left(\eta_{, l_{k}}^{i} l_{j}-\varepsilon_{j} \varepsilon_{k} \eta_{l_{j}}^{i} l_{k}\right) h_{k j}-\phi^{i j} l_{j}=0
$$

Since $\phi^{i j}$ depends only on $x, l, h_{i j}$ and $h_{j i}$, we obtain from (A.13) the following system

$$
\begin{aligned}
& \eta_{, l_{k}}^{i} l_{j}-\varepsilon_{j} \varepsilon_{k} \eta_{, l_{j}}^{i} l_{k}=0, \\
& \eta_{, x_{j}}^{i}+\left(\eta_{l_{i}}^{i} l_{j}-\varepsilon_{i} \varepsilon_{j} \eta_{l_{j}}^{i} l_{i}-\xi_{, x_{j}}^{j} l_{j}-\eta^{j}\right) h_{i j}-\phi^{i j} l_{j}=0 .
\end{aligned}
$$


By solving the characteristic system for (A.14), we have that $n^{i}=n^{i}\left(x, l_{i}\right)$. By taking derivatives of (A.15) with respect to $h_{j i}$ we get

$$
\phi_{, h_{j i}}^{i j}=0 .
$$

On the other hand, by taking the derivatives of (A.15) twice with respect to $h_{i j}$, we obtain

$$
\phi_{, h_{i j} h_{i j}}^{i j}=0
$$

Consequently, it follows from (A.16) and (A.17) that $\phi^{i j}$ is given by

$$
\phi^{i j}=A^{i j}(x, l) h_{i j}+B^{i j}(x, l) .
$$

Therefore, (A.6) reduces to

$$
\phi_{, x_{k}}^{i j}+\sum_{r} \phi_{, l_{r}}^{i j} l_{r, x_{k}}+A^{i j} h_{i j, x_{k}}-\xi_{, x_{k}}^{k} h_{i j, x_{k}}-\left(A^{i k} h_{i k}+B^{i k}\right) h_{k j}-\left(A^{k j} h_{k j}+B^{k j}\right) h_{i k}=0 .
$$

By considering the substitutions (A.1)-(A.5), this equation reduces to

$$
\begin{aligned}
\phi_{, x_{k}}^{i j} & +\phi_{, l_{i}}^{i j} h_{i k} l_{k}+\phi_{, l_{j}}^{i j} h_{j k} l_{k}-\phi_{, l_{k}}^{i j}\left(\varepsilon_{k} \varepsilon_{j} h_{j k} l_{j}+\varepsilon_{k} \varepsilon_{i} h_{i k} l_{i}\right) \\
& +A^{i j} h_{i k} h_{k j}-\xi_{, x_{k}}^{k} h_{i k} h_{k j}-A^{i k} h_{i k} h_{k j}-B^{i k} h_{k j}-A^{k j} h_{i k} h_{k j}-B^{k j} h_{i k}=0,
\end{aligned}
$$

which can be rewritten as

$$
\begin{aligned}
B_{, x_{k}}^{i j} & +A_{, x_{k}}^{i j} h_{i j}+\left(B_{, l_{i}}^{i j} l_{k}-\varepsilon_{k} \varepsilon_{i} B_{, l_{k}}^{i j} l_{i}-B^{k j}\right) h_{i k}+\left(B_{, l_{j}}^{i j} l_{k}-\varepsilon_{k} \varepsilon_{j} B_{, l_{k}}^{i j} l_{j}\right) h_{j k} \\
& -B^{i k} h_{k j}+\left(A_{l_{i}}^{i j} l_{k}-\varepsilon_{k} \varepsilon_{i} A_{, l_{k}}^{i j} l_{i}\right) h_{i k} h_{i j}+\left(A_{,_{j}}^{i j} l_{k}-\varepsilon_{k} \varepsilon_{j} A_{l_{k}}^{i j} l_{j}\right) h_{i j} h_{j k} \\
& +\left(A^{i j}-\xi_{, x_{k}}^{k}-A^{i k}-A^{k j}\right) h_{i k} h_{k j}=0 .
\end{aligned}
$$

It follows from the coefficients of $h_{k j}$ that $B^{i k}=0$. The permutation of the indices $i, j$ and $k$ leads to

$$
B^{s t}=0, \quad \forall s, t, \quad s \neq t .
$$

By equating to zero the coefficients of $h_{i k} h_{k j}$ and $h_{i j} h_{j k}$, the following system is obtained

$$
A_{, l_{i}}^{i j} l_{k}-\varepsilon_{k} \varepsilon_{i} A_{, l_{k}}^{i j} l_{i}=0, \quad A_{, l_{j}}^{i j} l_{k}-\varepsilon_{k} \varepsilon_{j} A_{, l_{k}}^{i j} l_{j}=0,
$$

where we solve the characteristic system to conclude that $A^{i j}$ depends only on $x$. On the other hand, the coefficient of $h_{i j}$ implies that $A^{i j}$ does not depend on $x_{k}$, therefore $A^{i j}=A^{i j}\left(x_{i}, x_{j}\right)$. Considering the coefficient of $h_{i k} h_{k j}$, we obtain the following equation

$$
A^{i j}-\xi_{, x_{k}}^{k}-A^{i k}-A^{k j}=0 .
$$

Therefore, equation (A.13) reduces to

$$
\eta_{, x_{j}}^{i}+\left(\eta_{, l_{i}}^{i} l_{j}-\xi_{, x_{j}}^{j} l_{j}-\eta^{j}-A^{i j} l_{j}\right) h_{i j}=0 .
$$

Since $\eta^{i}$ does not depend on $h_{i j}$, we must have

$$
\begin{aligned}
& \eta_{, x_{j}}^{i}=0 \\
& \eta_{, l_{i}}^{i} l_{j}-\xi_{, x_{j}}^{j} l_{j}-\eta^{j}-A^{i j} l_{j}=0
\end{aligned}
$$



that

By applying $\operatorname{pr}^{(1)} V$ to equation (3.10), we have $\phi_{(j)}^{i j}+\phi_{(i)}^{j i}+\phi^{i k} h_{k j}+h_{i k} \phi^{k j}=0$, which implies

$$
\phi_{, x_{j}}^{i j}+\phi_{, h_{i j}}^{i j} h_{i j, x_{j}}-\xi_{, x_{j}}^{j} h_{i j, x_{j}}+\phi_{, x_{i}}^{j i}+\phi_{, h_{j i}}^{j i} h_{j i, x_{i}}-\xi_{, x_{i}}^{i} h_{j i, x_{i}}++\phi^{i k} h_{k j}+h_{i k} \phi^{k j}=0 .
$$

Considering the substitution (A.4), for $i<j$, we obtain

$$
\phi_{, x_{j}}^{i j}+\phi_{, x_{i}}^{j i}+\left(\xi_{, x_{j}}^{j}-A^{i j}+A^{j i}-\xi_{, x_{i}}^{i}\right) h_{j i, x_{i}}+\left(\xi_{, x_{j}}^{j}-A^{i j}+A^{i k}+A^{k j}\right) h_{i k} h_{j k}=0 .
$$

Then, the coefficient of $h_{j i, x_{i}}$ leads to

$$
\xi_{, x_{j}}^{j}-A^{i j}+A^{j i}-\xi_{, x_{i}}^{i}=0 .
$$

By applying the prolongation $\operatorname{pr}^{(1)} V$ to (3.11) we have $\varepsilon_{i} \phi_{(i)}^{i j}+\varepsilon_{j} \phi_{(j)}^{j i}+\varepsilon_{k} \phi^{k i} h_{k j}+\varepsilon_{k} \phi^{k j} h_{k i}=0$, which implies

$$
\begin{aligned}
& \varepsilon_{i}\left(\phi_{, x_{i}}^{i j}+\phi_{, h_{i j}}^{i j} h_{i j, x_{i}}-\xi_{, x_{i}}^{i} h_{i j, x_{i}}\right)+\varepsilon_{j}\left(\phi_{, x_{j}}^{j i}+\phi_{, h_{j i}}^{j i} h_{j i, x_{j}}-\xi_{, x_{j}}^{j} h_{j i, x_{j}}\right) \\
& \quad+\varepsilon_{k}\left(\phi^{k i} h_{k j}+\phi^{k j} h_{k i}\right)=0 .
\end{aligned}
$$

Considering the substitution (A.5) with $i<j$, we obtain

$$
\varepsilon_{i} \phi_{, x_{j}}^{i j}+\varepsilon_{j} \phi_{, x_{j}}^{j i}+\varepsilon_{j}\left(\xi_{, x_{i}}^{i}-A^{i j}+A^{j i}-\xi_{, x_{j}}^{j}\right) h_{j i, x_{j}}+\varepsilon_{k}\left(\xi_{, x_{i}}^{i}-A^{i j}+A^{k i}+A^{k j}\right) h_{k i} h_{k j}=0 .
$$

From the coefficient of $h_{j i, x_{j}}$, we get

$$
A^{i j}-\xi_{, x_{i}}^{i}-A^{j i}+\xi_{, x_{j}}^{j}=0 .
$$

Therefore, it follows from (A.22) and (A.23) that

$$
A^{j i}=A^{i j} .
$$

Consequently, both equations imply that $\xi_{, x_{i}}^{i}=\xi_{, x_{j}}^{j}$, which enables us to conclude that

$$
\xi^{m}=a x_{m}+a_{m}, \quad \forall 1 \leq m \leq 3,
$$

where $a$ and $a_{m}$ are real constants. Moreover, from (A.25) and (A.20), we have that

$$
A^{i j}-a-A^{i k}-A^{k j}=0 \quad \text { and } \quad A^{i k}-a-A^{i j}-A^{j k}=0 .
$$

By taking the sum of these equations and using (A.24), we obtain $A^{k j}=-a$. Therefore, it follows from (A.18) and (A.19), that

$$
\phi^{s t}=-a h_{s t}, \quad \forall s \neq t .
$$

Moreover, from (A.25) and (A.21), we get

$$
\eta_{, l_{i}}^{i} l_{j}=\eta^{j}
$$

Since the function $\eta^{m}$ depends only on $x_{m}$ and $l_{m}$, we conclude that, $\eta_{, l_{i} l_{i}}^{i}=0$, i.e.,

$$
\eta^{i}=N^{i}\left(x_{i}\right) l_{i}+M^{i}\left(x_{i}\right)
$$

Hence, it follows from (A.27) and (A.28) that $\eta_{, l_{i}}^{i}=\eta_{, l_{j}}^{j}=N\left(x_{i}\right)$. Therefore, $N^{\prime}\left(x_{i}\right)=\eta_{, l_{j} x_{i}}^{j}=0$, which implies that,

$$
\eta^{i}=c l_{i}+M^{i}\left(x_{i}\right)
$$


Finally, we apply the prolongation $\operatorname{pr}^{(1)} V$ to equation (3.8) to obtain

$$
\varepsilon_{i} \eta_{(i)}^{i}+\varepsilon_{j} \phi^{j i} l_{j}+\varepsilon_{j} h_{j i} \eta^{j}+\varepsilon_{k} \phi^{k i} l_{k}+\varepsilon_{k} h_{k i} \eta^{k}=0
$$

which implies that

$$
\varepsilon_{i} \eta_{, x_{i}}^{i}+\varepsilon_{i} \eta_{, l_{i}}^{i} l_{i, x_{i}}-\varepsilon_{i} \xi_{, x_{i}}^{i} l_{i, x_{i}}+\varepsilon_{j} \phi^{j i} l_{j}+\varepsilon_{j} h_{j i} \eta^{j}+\varepsilon_{k} \phi^{k i} l_{k}+\varepsilon_{k} h_{k i} \eta^{k}=0 .
$$

When we substitute (A.2) for $l_{i, x_{i}}$ and we consider equations (A.25), (A.26) and (A.29), we obtain

$$
\varepsilon_{i} M_{, x_{i}}^{i}+\varepsilon_{j} h_{j i} M^{i}+\varepsilon_{k} h_{k i} M^{k}=0
$$

The analysis of the coefficients of $h_{j i}$ and $h_{k i}$ enables us to conclude that $M^{i}=M^{k}=0$, consequently, $\eta^{m}=c l_{m}, \forall 1 \leq m \leq 3$. This concludes the proof of Theorem 2 .

\section{Acknowledgements}

The authors were partially supported by CAPES/PROCAD and CNPq.

\section{References}

[1] Barbosa J.L., Ferreira W., Tenenblat K., Submanifolds of constant sectional curvature in pseudo-Riemannian manifolds, Ann. Global Anal. Geom. 14 (1996), 381-401.

[2] Cartan E., La déformation des hypersurfaces dans l'espace conforme réel à $n \geq 5$ dimensions, Bull. Soc. Math. France 45 (1917), 57-121.

[3] Corro A.V., Martínez A., Milán F., Complete flat surfaces with two isolated singularities in hyperbolic 3-space, J. Math. Anal. Appl. 366 (2010), 582-592, arXiv:0905.2371.

[4] Ferreira W., Soluções invariantes pelos grupos de simetria de Lie das Equações Generalizadas Intrínsecas de Laplace e de sinh-Gordon elíptica e propriedades geométricas das subvariedades associadas, Ph.D. thesis, Universidade de Brasília, 1994.

[5] Gálvez J.A., Martínez A., Milán F., Flat surfaces in the hyperbolic 3-space, Math. Ann. 316 (2000), 419-435.

[6] Guichard C., Sur les systèmes triplement indéterminés et sur les systèmes triplement orthogonaux, GauthierVillars, Paris, 1905.

[7] Hertrich-Jeromin U., Introduction to Möbius differential geometry, London Mathematical Society Lecture Note Series, Vol. 300, Cambridge University Press, Cambridge, 2003.

[8] Hertrich-Jeromin U., On conformally flat hypersurfaces and Guichard's nets, Beiträge Algebra Geom. 35 (1994), 315-331.

[9] Hertrich-Jeromin U., Suyama Y., Conformally flat hypersurfaces with Bianchi-type Guichard nets, Osaka J. Math., to appear.

[10] Hertrich-Jeromin U., Suyama Y., Conformally flat hypersurfaces with cyclic Guichard net, Internat. J. Math. 18 (2007), 301-329.

[11] Kokubu M., Rossman W., Saji K., Umehara M., Yamada K., Singularities of flat fronts in hyperbolic space, Pacific J. Math. 221 (2005), 303-351, math.DG/0401110.

[12] Kokubu M., Umehara M., Yamada K., Flat fronts in hyperbolic 3-space, Pacific J. Math. 216 (2004), 149-175, math.DG/0301224.

[13] Lafontaine J., Conformal geometry from the Riemannian viewpoint, in Conformal Geometry (Bonn, 1985/1986), Aspects Math., Vol. E12, Vieweg, Braunschweig, 1988, 65-92.

[14] Lamé G., Leçons sur les coordonnés curvilignes et leurs diverses applications, Mallet-Bachelier, Paris, 1859.

[15] Lie S., Theorie der Transformationsgruppen, B.G. Teubner, Leipzig, 1888, 1890, 1893.

[16] Martinez A., dos Santos J.P., Tenenblat K., Helicoidal flat surfaces in the hyperbolic 3-space, Pacific J. Math., to appear. 
[17] Olver P.J., Applications of Lie groups to differential equations, Graduate Texts in Mathematics, Vol. 107, Springer-Verlag, New York, 1986.

[18] Olver P.J., Symmetry groups and group invariant solutions of partial differential equations, J. Differential Geom. 14 (1979), 497-542.

[19] Rabelo M.L., Tenenblat K., Submanifolds of constant nonpositive curvature, Mat. Contemp. 1 (1991), 71-81.

[20] Suyama Y., Conformally flat hypersurfaces in Euclidean 4-space, Nagoya Math. J. 158 (2000), 1-42.

[21] Suyama Y., Conformally flat hypersurfaces in Euclidean 4-space. II, Osaka J. Math. 42 (2005), 573-598.

[22] Suyama Y., Conformally flat hypersurfaces in Euclidean 4-space and a class of Riemannian 3-manifolds, Sürikaisekikenkyūsho Kōkyūroku (2001), no. 1236, 60-89.

[23] Tenenblat K., Transformations of manifolds and applications to differential equations, Pitman Monographs and Surveys in Pure and Applied Mathematics, Vol. 93, Longman, Harlow, 1998.

[24] Tenenblat K., Winternitz P., On the symmetry groups of the intrinsic generalized wave and sine-Gordon equations, J. Math. Phys. 34 (1993), 3527-3542. 